\title{
RELATION LIFTING, WITH AN APPLICATION TO THE MANY-VALUED COVER MODALITY
}

\author{
MARTA BÍLKOVÁ $^{a}$, ALEXANDER KURZ $^{b}$, DANIELA PETRIŞAN ${ }^{c}$, AND JIŘÍ VELEBIL $^{d}$ \\ ${ }^{a}$ Institute of Computer Science, Academy of Sciences of the Czech Republic \\ e-mail address: bilkova@cs.cas.cz \\ ${ }^{b, c}$ Department of Computer Science, University of Leicester, United Kingdom \\ e-mail address: kurz@mcs.le.ac.uk, daniela.petrisan@gmail.com \\ ${ }^{d}$ Faculty of Electrical Engineering, Czech Technical University in Prague, Czech Republic \\ e-mail address: velebil@math.feld.cvut.cz
}

\begin{abstract}
We introduce basic notions and results about relation liftings on categories enriched in a commutative quantale. We derive two necessary and sufficient conditions for a 2 -functor $T$ to admit a functorial relation lifting: one is the existence of a distributive law of $T$ over the "powerset monad" on categories, one is the preservation by $T$ of "exactness" of certain squares. Both characterisations are generalisations of the "classical" results known for set functors: the first characterisation generalises the existence of a distributive law over the genuine powerset monad, the second generalises preservation of weak pullbacks.

The results presented in this paper enable us to compute predicate liftings of endofunctors of, for example, generalised (ultra)metric spaces. We illustrate this by studying the coalgebraic cover modality in this setting.
\end{abstract}

\section{INTRODUCTION}

Relation lifting [7, 15, 22] plays a crucial role in coalgebraic logic, see, e.g., [36, 5, 48]. On the one hand, it is used to explain bisimulation: If $T:$ Set $\longrightarrow$ Set is a functor, then the largest bisimulation on a coalgebra $\xi: X \longrightarrow T X$ is the largest fixed point of the operator $(\xi \times \xi)^{-1} \cdot \bar{T}$ on relations on $X$, where $\bar{T}$ is the lifting of $T$ from the category of sets and functions to the category of sets and relations. (The precise meaning of 'lifting' will be given in our setting as Definition 3.7.)

On the other hand, Moss's coalgebraic logic 36] is given by adding to propositional logic a modal operator $\nabla$, the semantics of which is given by applying $\bar{T}$ to the forcing relation $\Vdash \subseteq X \times \mathcal{L}$, where $\mathcal{L}$ is the set of formulas: If $\alpha \in T(\mathcal{L})$, then $x \Vdash \nabla \alpha \Leftrightarrow \xi(x) \bar{T}(\Vdash) \alpha$.

2012 ACM CCS: [Theory of computiation]: Logic-Modal and temporal logics.

2010 Mathematics Subject Classification: 18A15, 18A32.

Key words and phrases: Relation lifting, module, exact square, enriched categories, commutative quantale, coalgebra, modal logic, cover modality.

${ }^{a, d}$ Marta Bílková and Jiří Velebil acknowledge the support of the grant No. P202/11/1632 of the Czech Science Foundation. 
This paper presents a fundamental study of the relation lifting of a functor $T: \mathscr{Y}$-cat $\longrightarrow$ $\mathscr{V}$-cat, where $\mathscr{V}$ is a commutative quantale. Of particular importance to coalgebra is the case $\mathscr{V}=2$, the two-element chain. In that case, $\mathscr{Y}$-cat is the category Pre of preorders. In the same way as Set-coalgebras capture bisimulation, Pre-coalgebras (and Pos-coalgebras) capture simulation [40, 50, 24, 27, 32, 4]. This suggests that, in analogy with the Set-based case, a coalgebraic understanding of logics for simulations should derive from the study of Pos-functors together with on the one hand their predicate liftings and on the other hand their $\nabla$-operator. The beginnings of such a study where carried out in [10] and the purpose of this paper is to show how that work generalises from 2 -cat to $\mathscr{Y}$-cat, thus extending the scope of our work from coalgebras over preorders to coalgebras over generalised (ultra)metric spaces.

In addressing the problem of lifting a locally monotone endofunctor on the category of preorders/posets to an endofunctor of the category of monotone relations, [10] used the representation of monotone relations as certain spans, called two-sided discrete fibrations. That such a representation is possible is due to the fact that preorders can be viewed as small categories enriched in the two-element chain 2. Hence one works with discrete fibrations in the 2-category 2-cat and the above mentioned correspondence of monotone relations and two-sided discrete fibrations is manifested by the so-called Grothendieck construction.

The Grothendieck construction, however, is not available for a general (cocomplete, symmetric monoidal closed) base category $\mathscr{V}$, hence one cannot hope for the correspondence of "relations" in $\mathscr{K}$-cat and two-sided discrete fibrations. There is a remedy to this problem, that goes back to Ross Street [45]: the "relations" in $\mathscr{Y}$-cat correspond to the two-sided codiscrete cofibrations in $\mathscr{Y}$-cat, i.e., to two sided-discrete fibrations in $(\mathscr{Y} \text {-cat })^{o p}$.

For a general base category $\mathscr{V}$, a "relation"

$$
R: \mathscr{A} \longrightarrow \mathscr{B}
$$

from a $\mathscr{V}$-category $\mathscr{A}$ to a $\mathscr{V}$-category $\mathscr{B}$ is a $\mathscr{V}$-functor of the form

$$
R: \mathscr{B}^{o p} \otimes \mathscr{A} \longrightarrow \mathscr{V}
$$

called a module and it is represented by a cospan

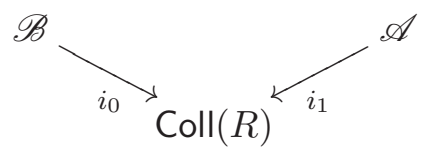

called the collage of $R$ that becomes a two-sided discrete fibration in ( $\mathscr{Y}$-cat $)^{o p}$.

Given a 2-functor $T: \mathscr{V}$-cat $\longrightarrow \mathscr{Y}$-cat, the desired relation lifting $\bar{T}: \mathscr{V}$-mod $\longrightarrow \mathscr{Y}$-mod, where by $\mathscr{V}$-mod we denote the 2 -category of $\mathscr{V}$-modules (= "relations"), is defined, on a module $R$ from $\mathscr{A}$ to $\mathscr{B}$, as follows:

Represent the module $R$ as the collage $\left(i_{0}, \operatorname{Coll}(R), i_{1}\right)$ and put $\bar{T}(R)$ to be the composite

$$
\mathscr{A} \stackrel{\left(T i_{1}\right)_{\diamond}}{\longrightarrow} \operatorname{Coll}(R) \stackrel{\left(T i_{0}\right)^{\diamond}}{\longrightarrow} \mathscr{B}
$$

of modules, where the upper and lower diamonds are certain canonical ways of making a functor into a module (the "graph" constructions).

Although one can define $\bar{T}$ in the above manner for any $T$, the resulting $\bar{T}$ will only be a lax functor. It extends $T$ iff $T$ preserves full and faithful 1-cells as shown by Worrell [50]. $\bar{T}$ being a lax relation lifting suffices to generalise the notion of (bi)simulation from Cat 
to $\mathscr{Y}$-cat. But for the applications to coalgebraic logic we need that $\bar{T}$ preserves identities and composition strictly, for which one needs to assume that $T: \mathscr{Y}$-cat $\longrightarrow \mathscr{Y}$-cat satisfies a certain condition that we call the Beck-Chevalley Condition (BCC).

Since we work with cospans rather than spans, we expect that the cospans corresponding to modules will be "jointly epimorphic" (just as the spans in sets, corresponding to binary relations, are jointly monomorphic). This is indeed the case: collages are (contained in) the "jointly epi" part of a factorisation system on $\mathscr{Y}$-cat that has fully faithful functors as the "mono part".

Hence the idea of composing two modules as cospans is as in

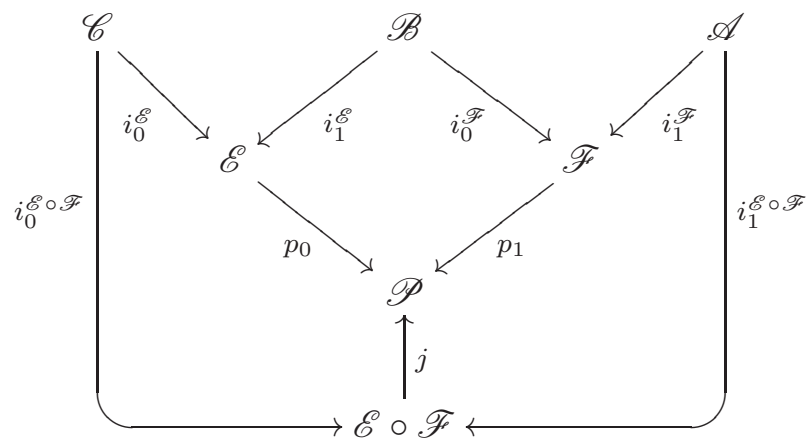

where the middle square is a pushout and $j: \mathscr{E} \circ \mathscr{F} \longrightarrow \mathscr{P}$ is the "mono part" of the factorisation of $\left[p_{0} i_{0}^{\mathscr{E}}, p_{1} i_{1}^{\mathscr{F}}\right]: \mathscr{C}+\mathscr{A} \longrightarrow \mathscr{P}$.

The Beck-Chevalley Condition for $T$ will then ensure that when applying $T$ to the above diagram and taking lower and upper diamonds where appropriate will yield that $\bar{T}(\mathscr{E} \circ \mathscr{F})=\bar{T}(\mathscr{E}) \cdot \bar{T}(\mathscr{F})$ holds.

The level of generality. Although all what follows, at least up to and including Section 5 . will work for a general complete and cocomplete base category $\mathscr{V}$, in view of applications and simplicity of exposition we confine ourselves to a particularly simple choice of base category $\mathscr{V}$, namely, a (complete) lattice.

Therefore, our results will enable us to compute predicate liftings of endofunctors of, for example, generalised (ultra)metric spaces.

The structure of the paper. We recall the basic facts about categories and functors enriched in a commutative quantale in Section 2. In Section 3 we argue that modules (in the sense of enriched category theory) are the proper generalisation of relations. We prove that the category of modules is a Kleisli category for a certain monad and derive the first (easy) characterisation of the existence of a relation lifting via the existence of a distributive law, see Corollary 3.14 below. For the second characterisation theorem one needs to analyse cospans that correspond to modules more in detail: this is done in the rest of Section 3. In Section 4 we prove that the cospans, corresponding to modules, are "jointly epi" w.r.t. a well-behaved factorisation system on $\mathscr{Y}$-cat. In fact, we prove that $(\mathscr{Y} \text {-cat })^{o p}$ becomes a "regular" category. Hence composition of relations (=modules) can be reduced to taking subobjects of pushouts of cospans. Having observed this, we analyse in Section 5 what it takes for a functor to preserve composition of cospans, arriving at the second characterisation theorem for a relation lifting in Corollary 5.11 below. Finally, we 
exhibit examples of various functors in Section 6 and indicate an application to coalgebraic logic in Section 7.

Related work. The basic categorical machinery on codiscrete cofibrations is contained in [45] and the idea of using factorisation systems for composition of collages is in [14, see also [39] for a more elementary treatment. The essential ideas about coregularity of $\mathscr{Y}$-cat w.r.t. codiscrete cofibrations appear in [37, we have included the proofs in our simpler setting for the sake of self-containedness. Coalgebras over a category enriched over a commutative quantale have been suggested for the study of simulations in [40] and studied in detail in [49, 50]. For the purpose of simulations, lax liftings are of interest and where [50] characterises lax liftings, we characterise non-lax (i.e., strict) liftings.

Acknowledgement. We thank the anonymous referees for their helpful suggestions and comments.

\section{The BASE CATEGORY $\mathscr{V}$}

We will enrich all our categories, functors, etc. in a particularly simple (co)complete symmetric monoidal closed base category $\mathscr{V}$. Namely, we assume that

$$
\mathscr{V}_{O}
$$

is a complete lattice with the lattice order written as $\leq$, the symbol $\perp$ denotes the least element and $T$ the greatest element of $\mathscr{V}_{O}$.

We further denote by $\otimes$ the symmetric monoidal structure on $\mathscr{V}_{o}$, having a unit element I. The closed structure (the internal hom) of $\mathscr{V}_{o}$ is denoted by $[x, y]$. Hence we have adjunction relations

for every $x, y, z$ in $\mathscr{V}_{o}$.

$$
x \otimes y \leq z \quad \text { iff } \quad y \leq[x, z]
$$

The whole structure as above is denoted by

$$
\mathscr{V}=\left(\mathscr{V}_{o}, \otimes, I,[-,-]\right)
$$

and it is sometimes called a commutative quantale.

Definition 2.1. A category $\mathscr{A}$ enriched in $\mathscr{V}$ (or, a $\mathscr{V}$-category) consists of the following data:

(1) A class of objects denoted by $a, b, \ldots$

(2) For every pair $a, b$ of objects a hom-object $\mathscr{A}(a, b)$ in $\mathscr{V}_{o}$.

The data are subject to the following axioms:

(1) For every object $a$ there is an inequality

$$
I \leq \mathscr{A}(a, a)
$$

witnessing the "choice of the identity morphism on $a$ ".

(2) For every triple $a, b, c$ of objects there is an inequality

$$
\mathscr{A}(b, c) \otimes \mathscr{A}(a, b) \leq \mathscr{A}(a, c)
$$

witnessing "the composition of morphisms". 
Notice that the other two usual axioms (identity morphisms are identities w.r.t. composition and composition is associative, see [25]) become void, since we enrich in a poset.

Observe that $\mathscr{V}$ itself becomes a $\mathscr{V}$-category by putting $\mathscr{V}(x, y)=[x, y]$.

Our previous work [10] was based on the following instance.

Example 2.2. $\mathscr{V}_{o}$ is the two-element chain 2, i.e., there are two objects 0 and 1 with $0 \leq 1$. The tensor in 2 is the meet and the internal hom is implication.

A $\mathscr{V}$-category is a (possibly large) preorder.

In this paper we are also interested in the following examples of $\mathscr{V}$, going back at least to 31 .

\section{Example 2.3.}

(1) $\mathscr{V}_{O}$ is the unit interval $[0 ; 1]$ with $\leq$ being the reversed order $\geq_{R}$ of the real numbers. The unit $I$ is 0 and $x \otimes y=\max \{x, y\}$ where the maximum is taken w.r.t. the usual order $\leq_{\mathrm{R}}$. The internal hom is given by $[0 ; 1](x, y)=$ if $x \geq_{\mathrm{R}} y$ then 0 else $y$.

A $\mathscr{V}$-category $\mathscr{A}$ is a (possibly large) generalised ultrametric space: the hom-object $\mathscr{A}(a, b)$ is the "distance" of $a$ and $b$ (notice that distance need not be symmetric), the axiom for identities becomes the requirement that $\mathscr{A}(a, a)=0$ holds for all $a$, and the axiom for composition is the ultrametric triangle inequality $\mathscr{A}(a, c) \leq_{\mathrm{R}} \max \{\mathscr{A}(a, b), \mathscr{A}(b, c)\}$ for all $a, b, c$.

(2) $\mathscr{V}_{o}$ is the interval $[0 ; \infty]$ with $\leq$ being the reversed order $\geq_{R}$ of the reals. Extend the usual addition of nonnegative reals by putting $x+\infty=\infty+x=\infty$, for every $x \in[0 ; \infty]$ and let $x \otimes y=x+y$, the unit $I$ being 0 . The internal hom is given by truncated subtraction $[0 ; 1](x, y)=y-x=$ if $x \geq_{\mathrm{R}} y$ then 0 else $y-x$.

A $\mathscr{V}$-category $\mathscr{A}$ is a (possibly large) generalised metric space: the hom-object $\mathscr{A}(a, b)$ is the "distance" of $a$ and $b$ (notice that distance need not be symmetric and it may be infinite), the axiom for identities becomes the requirement that $\mathscr{A}(a, a)=0$ holds for all $a$, and the axiom for composition is the triangle inequality $\mathscr{A}(a, c) \leq_{\mathrm{R}}$ $\mathscr{A}(a, b)+\mathscr{A}(b, c)$ for all $a, b, c$.

(3) An example of a quantale $\mathscr{V}$ that leads to probabilistic metric spaces is the following one: let $\mathscr{V}_{o}$ be the poset of all functions $f:[0 ; \infty] \longrightarrow[0 ; 1]$ such that $f(x)=\bigvee_{y<x} f(y)$ with the pointwise order. By defining

$$
f \otimes g(z)=\bigvee_{x+y \leq z} f(x) \cdot g(y)
$$

we obtain a quantale $\mathscr{V}$. A $\mathscr{V}$-category $\mathscr{A}$ is a (generalised) probabilistic metric space: for every pair $a, a^{\prime}$ of objects of $\mathscr{A}$, the hom-object is a function

$$
\mathscr{A}\left(a, a^{\prime}\right):[0 ; \infty] \longrightarrow[0 ; 1]
$$

with the intuitive meaning $\mathscr{A}\left(a, a^{\prime}\right)(r)=s$ holds iff $s$ is the probability that the distance from $a$ to $a^{\prime}$ is smaller than $r$. See [23] and [18].

The first two items above have been studied extensively in [11] and [12], respectively. They should serve as a source of intuition: if $\mathscr{V}$ is a commutative quantale, then $\mathscr{V}$-categories should be thought of as "generalised metric spaces with the metric valued in $\mathscr{V}_{o}$ ", see [18] for more in this direction. The following example illustrating both items above and the use of a non-symmetric metric is taken from [11, 12]. 
Example 2.4. Given a set $A$, we can turn the set $A^{\infty}$ of finite and infinite words $v=v_{1} v_{2} \ldots$ with $v_{i} \in A$ into a non-symmetric generalised (ultra)metric space. Define $A^{\infty}(v, w)=0$ if $v$ is a prefix of $w$ and $A^{\infty}(v, w)=2^{-n}$ otherwise where $n \in \mathbb{N}$ is the length of the longest common prefix of $v$ and $w$.

Another source of examples and intuitions comes from many-valued or fuzzy logic [20]:

Example 2.5. There are $\mathscr{V}$ 's coming from the theory of fuzzy sets: suppose that $I=\top$ is the top element of $\mathscr{V}_{o}$, then a small $\mathscr{V}$-category $\mathscr{A}$ consists of a set of objects and the hom-objects satisfy the axioms

$$
\mathscr{A}(a, a)=\top, \quad \mathscr{A}\left(a, a^{\prime}\right) \otimes \mathscr{A}\left(a^{\prime}, a^{\prime \prime}\right) \leq \mathscr{A}\left(a, a^{\prime \prime}\right)
$$

If, moreover, $\mathscr{A}\left(a, a^{\prime}\right)=\mathscr{A}\left(a^{\prime}, a\right)$ holds for all $a$ and $a^{\prime}$, then to give a small $\mathscr{V}$-category $\mathscr{A}$ is to give a 'similarity relation' on the set of objects of $\mathscr{A}$.

Examples of $\mathscr{V}$ 's:

(1) The unit interval $[0 ; 1]$ with the usual order and the Eukasiewicz tensor $x \otimes y=\max \{x+$ $y-1,0\}$. The internal hom is given by $[x, y]=$ if $x \leq y$ then 1 else $1-x+y$.

(2) The unit interval $[0 ; 1]$ with the usual order and the Gödel tensor $x \otimes y=\min \{x, y\}$. The internal hom is given by $[x, y]=$ if $x \leq y$ then 1 else $y$.

(3) The unit interval [0;1] with the usual order and the product tensor $x \otimes y=x \cdot y$. The internal hom is given by $[x, y]=$ if $x \leq y$ then 1 else $\frac{y}{x}$.

(4) The chain $\perp=x_{0}<x_{1}<\cdots<x_{n}=\top$ for some natural number $n$, with the Łukasiewicz tensor $x_{i} \otimes x_{j}=x_{\max \{i+j-n, 0\}}$.

(5) The chain $\perp=x_{0}<x_{1}<\cdots<x_{n}=\top$ for some natural number $n$, with the Gödel tensor $x_{i} \otimes x_{j}=x_{\min \{i, j\}}$.

Remark 2.6. Examples 2.3 and 2.5 are closely related. In fuzzy logic, binary operators that have an adjoint are called t-norms, in category theory they are called monoidal structures. For example, $x \mapsto 1-x$ is an isomorphism between Example 2.3(2) and Example 2.5(2).

Notation 2.7. For any $\mathscr{V}$-category $\mathscr{A}$ we denote by $\mathscr{A}^{o p}$ its opposite $\mathscr{V}$-category. $\mathscr{A}^{\text {op }}$ has the same objects as $\mathscr{A}$ and the equality

$$
\mathscr{A}^{o p}(a, b)=\mathscr{A}(b, a)
$$

holds for all $a$ and $b$.

For two $\mathscr{V}$-categories $\mathscr{A}$ and $\mathscr{B}$ we denote by

$$
\mathscr{A} \otimes \mathscr{B}
$$

the $\mathscr{V}$-category having pairs $(a, b)$ as objects and

$$
\mathscr{A} \otimes \mathscr{B}\left((a, b),\left(a^{\prime}, b^{\prime}\right)\right)=\mathscr{A}\left(a, a^{\prime}\right) \otimes \mathscr{B}\left(b, b^{\prime}\right)
$$

and we call it a tensor product of $\mathscr{A}$ and $\mathscr{B}$.

Remark 2.8. As always, every $\mathscr{V}$-category $\mathscr{A}$ has its underlying ordinary category $\mathscr{A}_{0}$ defined as follows:

(1) Objects of $\mathscr{A}_{O}$ are the same as objects of $\mathscr{A}$.

(2) There is a morphism $a \longrightarrow b$ in $\mathscr{A}_{0}$ iff the inequality $I \leq \mathscr{A}(a, b)$ holds.

Observe that an underlying category $\mathscr{A}_{0}$ is always a (possibly large) preorder. Furthermore, the underlying ordinary category of $\mathscr{V}$ is the lattice $\mathscr{V}_{o}$ (seen as a category in the usual sense). Conversely, any (possibly large) preorder $\langle A, \sqsubseteq\rangle$ gives rise to a free $\mathscr{V}$-category $\mathscr{A}$ having elements of $A$ as objects and $\mathscr{A}\left(a, a^{\prime}\right)=I$ if $a \sqsubseteq a^{\prime}$ and $\mathscr{A}\left(a, a^{\prime}\right)=\perp$ otherwise. 
Example 2.9. Going back to the generalised metric spaces of Example 2.3, we find that there is a morphism $a \longrightarrow b$ in $\mathscr{A}_{O}$ iff $\mathscr{A}(a, b)=0$, so that we have

$$
a \leq b \Leftrightarrow \mathscr{A}(a, b)=0 \text {. }
$$

In particular, for $A^{\infty}$ as in Example 2.4, we have that $\leq$ coincides with the prefix order.

Definition 2.10. Given $\mathscr{V}$-categories $\mathscr{A}, \mathscr{B}$, a $\mathscr{V}$-functor $f: \mathscr{A} \longrightarrow \mathscr{B}$ is given by the following data:

(1) An object assignment: for every object $a$ in $\mathscr{A}$, there is a unique object $f a$ in $\mathscr{B}$.

(2) An action on hom-objects: for every pair $a, a^{\prime}$ of objects of $\mathscr{A}$ there is an inequality

$$
\mathscr{A}\left(a, a^{\prime}\right) \leq \mathscr{B}\left(f a, f a^{\prime}\right)
$$

in $\mathscr{V}_{0}$.

Again, due to enrichment in a poset, the two requirements (preservation of identities and composition) become void. See [25].

Example 2.11. For every $\mathscr{V}$-category, the hom-object assignment $(a, b) \mapsto \mathscr{A}(a, b)$ gives rise to the hom-functor

$$
\mathscr{A}: \mathscr{A}^{o p} \otimes \mathscr{A} \longrightarrow \mathscr{V}
$$

Example 2.12. Observe that 2-functors are exactly the monotone maps between preorders. $\mathscr{V}$-functors for $\mathscr{V}=[0 ; 1]$ or $\mathscr{V}=[0 ; \infty]$ as in Example 2.3 are exactly the nonexpanding maps, that is, maps $f: \mathscr{A} \longrightarrow \mathscr{B}$ such that $\mathscr{B}\left(f a, f a^{\prime}\right) \leq_{\mathrm{R}} \mathscr{A}\left(a, a^{\prime}\right)$ holds for all $a, a^{\prime}$ in $\mathscr{A}$.

Notation 2.13. We denote by

$$
[\mathscr{A}, \mathscr{B}]
$$

the $\mathscr{V}$-category of $\mathscr{V}$-functors and $\mathscr{V}$-natural transformations, where we put

$$
[\mathscr{A}, \mathscr{B}](f, g)=\bigwedge_{a} \mathscr{B}(f a, g a)
$$

Remark 2.14. Given $\mathscr{V}$-functors $f: \mathscr{A} \longrightarrow \mathscr{B}$ and $g: \mathscr{A} \longrightarrow \mathscr{B}, \mathscr{V}$-natural transformations correspond to arrows $I \longrightarrow[\mathscr{A}, \mathscr{B}](f, g)$. If $\mathscr{V}$ is a poset, this means that there is a $\mathscr{V}$-natural transformation iff the inequality

$$
I \leq \bigwedge_{a} \mathscr{B}(f a, g a)
$$

holds in $\mathscr{V}_{o}$, in which case we write

$$
f \leq g .
$$

$f \leq g$ means exactly that $f a \leq g a$ holds in $\mathscr{B}_{o}$ for every $a$ in $\mathscr{A}$.

Example 2.15. Particularly important functor categories are the categories $[\mathscr{A}, \mathscr{V}]$ of $\mathscr{V}$ valued predicates over $\mathscr{A}$. Note that $\mathscr{V}$ as a complete lattice has some logical structure, with conjunctions corresponding to meets and disjunctions to joins. We also write true and false for the top and the bottom element, respectively.

(1) In case that $\mathscr{V}=2$, we we can identify $\varphi, \psi: \mathscr{A} \longrightarrow 2$ with up-sets $\bar{\varphi}, \bar{\psi}$ and then we have that $[\mathscr{A}, 2](\varphi, \psi)=1$ iff $\varphi(a) \leq \psi(a)$ for all $a$ in $A$ iff $\bar{\varphi} \subseteq \bar{\psi}$. 
(2) In the generalised metric spaces of Example 2.3, we have that functors $\varphi, \psi: \mathscr{A} \longrightarrow \mathscr{V}$ are many-valued predicates. We have true $=0$ and false $=1$ in Example 2.3.1 and false $=\infty$ in Example 2.3.2. We sometimes think of the value $\varphi(a)$ as the distance of " $a$ satisfies $\varphi$ " from being true, or the cost of turning " $a$ satisfies $\varphi$ " into a true statement. In the particular case of $A^{\infty}$ as in Example 2.4, examples include $\varphi_{\square a}, \varphi_{\diamond a}: A^{\infty} \longrightarrow \mathscr{V}$ for some fixed $a \in A$ defined by

$\varphi_{\square a}(v)=1-\sum_{n=1}^{\infty} 2^{-n} \cdot v a(n) \quad$ where $v a(n)=1$ if $v_{n}=a$ and $v a(n)=0$ otherwise

$\varphi \diamond a(v)= \begin{cases}1 & \text { if } a \text { does not occur in } v \\ 1-2^{-n} & \text { if } n \text { is the smallest number such that } v_{n+1}=a\end{cases}$

Intuitively, $\varphi_{\square a}$ means 'always $a$ ' with $\varphi_{\square a}(v)=$ true iff $v=a^{\omega}$ is the infinite string consisting only of $a$ 's; $\varphi_{\diamond a}$ means 'sometimes $a$ ' with $\varphi_{\diamond a}(v)=$ true iff the first element of $v$ is $a$ and the value $\varphi_{\diamond a}(v)$ decaying the longer fulfilling the 'promise of $a$ ' is postponed. On the other hand maps $\varphi_{\square \diamond a}, \varphi_{\diamond \square a}: \mathscr{A}_{O} \longrightarrow \mathscr{V}$ satisfying

$$
\begin{array}{ll}
\varphi_{\square \diamond a}(v)=0 & \text { iff } v \text { contains infinitely many } a \text { 's. } \\
\varphi \diamond \square a(v)=0 & \text { iff } v \text { has an infinite suffix consisting only of } a \text { 's. }
\end{array}
$$

cannot be made functorial (non-expansive). Indeed, let $\varphi$ be any of the two above. Then $\varphi\left(b^{\omega}\right)>_{\mathrm{R}} 0$. It follows $A^{\infty}\left(b^{n} a^{\omega}, b^{\omega}\right)=2^{-n}<_{\mathrm{R}} \varphi\left(b^{\omega}\right)=\mathscr{V}\left(\varphi\left(b^{n} a^{\omega}\right), \varphi\left(b^{\omega}\right)\right)$ for some large enough $n$, violating non-expansiveness. Intuitively, these properties $\varphi$ fail to be non-expansive because any prefix of finite length can be extended to an infinite word $v$ with $\varphi(v)=$ true.

The order on predicates induced by $\mathscr{V}$-natural transformations is implication. For example we do have

$$
\text { false } \leq \varphi_{\square a} \leq \varphi_{\diamond a} \leq \text { true }
$$

Indeed, $[\mathscr{A}, \mathscr{V}](\varphi, \psi)=\bigwedge_{a}[\varphi(a), \psi(a)]=\sup \{\mathscr{V}(\varphi(a), \psi(a)) \mid a \in \mathscr{A}\}$ so that we have $\varphi \leq \psi \Leftrightarrow \sup \{\mathscr{V}(\varphi(a), \psi(a)) \mid a \in \mathscr{A}\}=0$ where sup refers to the order of the reals.

It follows from Remark 2.14 that every $[\mathscr{A}, \mathscr{B}]$, as any $\mathscr{V}$-category, is a preorder and we denote by

$$
\mathscr{N} \text {-cat }
$$

the Pre-category of small $\mathscr{V}$-categories, $\mathscr{V}$-functors and $\mathscr{V}$-natural transformations.

Remark 2.16. That $\mathscr{Y}$-cat is a Pre-category will simplify our proofs: we will not need to worry about coherence conditions, since they will be satisfied automatically.

Remark 2.17. We omit the prefix $\mathscr{V}$ - when speaking about individual $\mathscr{V}$-categories and $\mathscr{V}$-functors in what follows.

\section{Example 2.18.}

(1) For $\mathscr{V}=2, \mathscr{V}$-cat is the Pre-category Pre of preorders, and monotone maps that are ordered pointwise. This Pre-category has been studied in [10] and here we are going to generalise this to also cover the next examples.

(2) For $\mathscr{V}=[0 ; 1]$ as in Example 2.3, $\mathscr{Y}$-cat is the Pre-category GUlt of generalised ultrametric spaces and nonexpanding maps. For $f, g: \mathscr{A} \longrightarrow \mathscr{V}$ we have $f \leq g \Leftrightarrow f a \geq_{\mathrm{R}} g a$ for all $a$. 
(3) For $\mathscr{V}=[0 ; \infty]$ as in Example 2.3. $\mathscr{Y}$-cat is the Pre-category GMet of generalised metric spaces and nonexpanding maps. For $f, g: \mathscr{A} \longrightarrow \mathscr{V}$ we have $f \leq g \Leftrightarrow f a \geq_{\mathrm{R}} g a$ for all $a$.

We finish by some examples of generalised metric spaces.

\section{Example 2.19.}

(1) Given a set $A$, we can turn the set $\mathscr{A}^{\mathrm{N}}$ of infinite words (or streams) over $A$ into an ultrametric space with the metric $A^{\mathbb{N}}(v, w)=2^{-n}$ where $n \in \mathbb{N}$ is the length of the largest common prefix of $v$ and $w$. This space is symmetric and well-known in metric domain theory [6].

(2) A non-symmetric version of the space above has been given as Example 2.4.

(3) A variant on item (2) above which does not satisfy the stronger triangle inequality of ultrametric spaces but only the triangle inequality of metric spaces goes as follows. Writing $v \wedge w$ for the longest common prefix of $v, w$ and $|v|$ for the length of $v$, we define $\mathscr{A}^{\infty}(v, w)=\sum_{i=|v \wedge w|+1}^{|v|} 2^{-i}$. In particular, $\mathscr{A}^{\infty}(v, w)=0$ if $v$ is a prefix of $w$ and $\mathscr{A}^{\infty}(v, w)=2^{-|v \wedge w|}$ if $v$ is infinite and not a prefix of $w$. Intuitively, $\mathscr{A}^{\infty}(v, w)$ measures the cost of 'building up' $v$ from $w$ if the cost of adding a letter at stage $i$ is $2^{-i}$.

(4) Let $\mathscr{A}$ be in GMet. Then $\mathscr{A}^{\mathrm{N}}=[\mathbb{N}, \mathscr{A}]$ has as carrier the set $\mathscr{A}_{0}^{\mathrm{N}}$ of infinite words over $\mathscr{A}$ and metric $\mathscr{A}^{\mathbb{N}}(v, w)=\sup \left\{\mathscr{A}\left(v_{n}, w_{n}\right) \mid n \in \mathbb{N}\right\}$.

(5) If distances in $\mathscr{A}$ are bounded by 1 , then $\mathscr{A}_{0}^{\mathrm{N}}$ can be equipped with a different metric $\mathscr{A}^{\mathbb{N}}(v, w)=\sum_{n \in \mathbb{N}} 2^{-n} \cdot \mathscr{A}\left(v_{n}, w_{n}\right)$. Intuitively, and differently to the previous examples, for two infinite words $v, w$, the index $i$ contributes to the cost $\mathscr{A}^{\mathrm{N}}(v, w)$ only if $v_{i} \neq w_{i}$.

\section{The MOdules}

A relation from $A$ to $B$ in Set can be seen as a map $B \times A \longrightarrow\{0,1\}$. Similarly, a monotone relation from $\mathscr{A}$ to $\mathscr{B}$ in Pre is a monotone function $\mathscr{B}^{o p} \times \mathscr{A} \longrightarrow 2$ and this was the setting of [10]. Here, we generalise to $\mathscr{B}^{o p} \otimes \mathscr{A} \longrightarrow \mathscr{V}$, which are now called modules, following standard terminology [45].

We recall in this section that modules constitute a category $\mathscr{V}$-mod, enriched in the category Pos of posets. Moreover we recall that there is a natural "graph" functor $(-)_{\diamond}$ : $\mathscr{V}$-cat $\longrightarrow \mathscr{Y}$-mod that will enable us to formulate the problem of a relation lifting. We also prove an easy solution of the relation lifting problem: since $\mathscr{K}$-mod is a Kleisli category of a certain monad on $\mathscr{V}$-cat, to have a relation lifting is to admit a distributive law over that certain monad, see Corollary 3.14 below. At the end of this section we also recall the nature of cospans that correspond to modules.

For more details on modules and (co)fibrations, see Street's paper [45. Our Corollary 3.14 is a slight generalisation of results in 35.

Definition 3.1. A module from $\mathscr{A}$ to $\mathscr{B}$, denoted by

$$
R: \mathscr{A} \longrightarrow \mathscr{B}
$$

is a functor $R: \mathscr{B}^{o p} \otimes \mathscr{A} \longrightarrow \mathscr{V}$.

Given modules

$$
R: \mathscr{A} \longrightarrow \mathscr{B} \quad S: \mathscr{B} \longrightarrow \mathscr{C}
$$


we define their composite

$$
S \cdot R: \mathscr{A} \longrightarrow \mathscr{C}
$$

to be the functor with values

$$
S \cdot R(c, a)=\bigvee_{b} S(c, b) \otimes R(b, a)
$$

for all $c$ and $a$.

Example 3.2. If $\mathscr{V}$ as in Example 2.3 and $R: \mathscr{A} \longrightarrow \mathscr{B}$ then we can think of $R(b, a)$ as "the cost of going from $a$ to $b$ ". That $R$ is a module and not just a $\mathscr{V}$-valued relation on the underlying sets means precisely

$$
\begin{aligned}
& \mathscr{B}\left(b^{\prime}, b\right) \otimes R(b, a) \geq_{\mathrm{R}} R\left(b^{\prime}, a\right) \\
& R(b, a) \otimes \mathscr{A}\left(a, a^{\prime}\right) \geq_{\mathrm{R}} R\left(b, a^{\prime}\right)
\end{aligned}
$$

stating that "going directly is cheaper than making a detour". Here $\otimes$ is max or + depending on the choice of $\mathscr{V}$ in Example 2.3. The reader might wish to go over Examples 2.19 with $R$ being the internal hom.

It is easy to prove that the composition of modules is associative and that it has homfunctors $\mathscr{A}: \mathscr{A}^{o p} \otimes \mathscr{A} \longrightarrow \mathscr{V}$ as units.

Define module transformations from $S$ to $S^{\prime}$ to be the natural transformations between the respective functors.

Remark 3.3. Suppose $S, S^{\prime}: \mathscr{A} \longrightarrow \mathscr{B}$ are modules such that $S \leq S^{\prime}$ and $S^{\prime} \leq S$ hold. In other words: suppose the inequalities

$$
I \leq\left[S(b, a), S^{\prime}(b, a)\right] \quad \text { and } \quad I \leq\left[S^{\prime}(b, a), S(b, a)\right]
$$

hold in $\mathscr{V}_{o}$ for all $b$ and $a$. Due to the monoidal closedness of $\mathscr{V}$, the above inequalities hold if and only if the inequalities

$$
S(b, a) \leq S^{\prime}(b, a) \quad \text { and } \quad S^{\prime}(b, a) \leq S(b, a)
$$

hold in $\mathscr{V}_{o}$ for all $b$ and $a$. Hence the equality

$$
S(b, a)=S^{\prime}(b, a)
$$

holds for all $b$ and $a$, since $\mathscr{V}_{o}$ is a poset.

Therefore small categories, modules, and module transformations organise themselves in a Pos-category denoted by

$$
\mathscr{V} \text {-mod }
$$

Remark 3.4. That $\mathscr{Y}$-mod is a Pos-category will again simplify the reasoning: we need not to worry about coherence conditions and every isomorphism 2-cell in $\mathscr{V}$-mod is identity.

Definition 3.5. Given $f: \mathscr{A} \longrightarrow \mathscr{B}$ in $\mathscr{Y}$-cat, the module $f_{\diamond}: \mathscr{A} \longrightarrow \mathscr{B}$ given by

$$
f_{\diamond}(b, a)=\mathscr{B}(b, f a)
$$

is called the graph of $f$. 
Remark 3.6. It is standard to prove that every module $f_{\diamond}$ is a left adjoint in $\mathscr{Y}$-mod, having the module

$$
f^{\diamond}(a, b)=\mathscr{B}(f a, b)
$$

as a right adjoint. The unit $\eta^{f}$ of $f_{\diamond} \dashv f^{\diamond}$ is given by the inequality $\mathscr{A}\left(a, a^{\prime}\right) \leq \mathscr{B}\left(f a, f a^{\prime}\right)=$ $\bigvee_{b} \mathscr{B}(f a, b) \otimes \mathscr{B}\left(b, f a^{\prime}\right)$ and the counit $\varepsilon^{f}$ of $f_{\diamond} \dashv f^{\diamond}$ is given by the inequality $\bigvee_{a} \mathscr{B}(b, f a) \otimes$ $\mathscr{B}\left(f a, b^{\prime}\right) \leq \mathscr{B}\left(b, b^{\prime}\right)$.

It is straightforward to see that the assignment $f \mapsto f_{\diamond}$ defines a 2 -functor, called the graph 2-functor

$$
(-)_{\diamond}: \mathscr{Y} \text {-cat } \longrightarrow \mathscr{V} \text {-mod }
$$

On objects (= on categories), the 2 -functor $(-)_{\diamond}$ is the identity. The assignment $f \mapsto f_{\diamond}$ clearly preserves composition and identities. Further, if $f \leq g$, then $f_{\diamond} \leq g_{\diamond}$ holds.

We are going to lift 2-functors $T: \mathscr{H}$-cat $\longrightarrow \mathscr{Y}$-cat along the graph 2-functor. More precisely:

Definition 3.7. A relation lifting of a 2 -functor $T: \mathscr{V}$-cat $\longrightarrow \mathscr{V}$-cat is a 2 -functor $\bar{T}$ : $\mathscr{Y}$-mod $\longrightarrow \mathscr{Y}$-mod, making the square

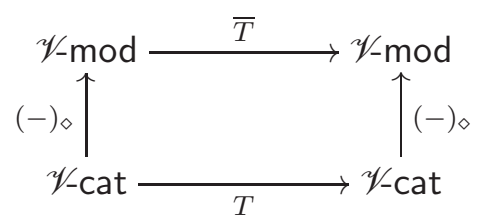

commutative.

We will characterise functors $T$ admitting a relation lifting in two ways, analogously to the "classical case":

(1) $T$ will have to distribute over a certain 2 -monad on $\mathscr{Y}$-cat.

In the classical case, $T:$ Set $\longrightarrow$ Set has a relation lifting iff $T$ distributes over the powerset monad on Set.

(2) $T$ will have to preserve certain squares in $\mathscr{V}$-cat.

In the classical case, $T$ : Set $\longrightarrow$ Set has a relation lifting iff $T$ preserves weak pullbacks. While the first characterisation will follow relatively easy (see the next part, Corollary 3.14), the second characterisation will require some technical work.

3.A. Modules as a Kleisli category. We prove that $(-)_{\diamond}: \mathscr{V}$-cat $\longrightarrow \mathscr{Y}$-mod has a right adjoint. The resulting 2 -monad $(\mathbb{L}, y, m)$ on $\mathscr{Y}$-cat will be proved to be analogous to the powerset monad on Set. Moreover, in the same way as the category of "classical" relations is a Kleisli category for the powerset monad on Set, the 2-category $\mathscr{V}$-mod will be the Kleisli category for the monad on $\mathscr{Y}$-cat. These results are easy modifications of the results for $\mathscr{V}=2$ from $[35]$.

Proposition 3.8. The 2-functor $(-)_{\diamond}: \mathscr{V}$-cat $\longrightarrow \mathscr{V}$-mod has a right adjoint 2-functor, denoted by

$$
(-)^{\dagger}: \mathscr{V} \text {-mod } \longrightarrow \mathscr{V} \text {-cat }
$$

Moreover, the resulting adjunction $(-)_{\diamond} \dashv(-)^{\dagger}$ is of $K Z$ type. That is, for every category $\mathscr{A}$, there is an adjunction

$$
\left(\eta_{\mathscr{A}}\right)_{\diamond} \dashv \varepsilon_{\mathscr{A} \diamond}:\left(\left(\mathscr{A}_{\diamond}\right)^{\dagger}\right)_{\diamond} \longrightarrow \mathscr{A}_{\diamond}
$$


where $\eta$ and $\varepsilon$ denote the unit and counit of $(-)_{\diamond} \dashv(-)^{\dagger}$.

Proof. Recall that $(-)_{\diamond}$ is identity on objects, i.e., recall that $\mathscr{A}_{\diamond}=\mathscr{A}$, for every category $\mathscr{A}$.

To define the right adjoint $(-)^{\dagger}$, put $\mathscr{A}^{\dagger}=\left[\mathscr{A}^{o p}, \mathscr{V}\right]$ and define $R^{\dagger}:\left[\mathscr{A}^{o p}, \mathscr{V}\right] \longrightarrow$ $\left[\mathscr{B}^{o p}, \mathscr{V}\right]$ to be the left Kan extension of $a \mapsto R(-, a)$ along the Yoneda embedding $a \mapsto$ $\mathscr{A}(-, a)$. In a formula, for $f: \mathscr{A}^{o p} \longrightarrow \mathscr{V}$, we have

$$
R^{\dagger}(f): b \mapsto \bigvee_{a} f a \otimes R(b, a)
$$

The unit of $(-)_{\diamond} \dashv(-)^{\dagger}$ is given by the Yoneda embedding $y_{\mathscr{A}}: \mathscr{A} \longrightarrow\left[\mathscr{A}^{o p}, \mathscr{V}\right]$. The counit is the evaluation relation $\mathrm{ev}_{\mathscr{A}}:\left[\mathscr{A}^{o p}, \mathscr{V}\right] \longrightarrow \mathscr{A}$ with $\mathrm{ev}_{\mathscr{A}}(a, f)=f(a)$.

Observe that $\operatorname{ev}_{\mathscr{A}}(a, f)=\left[\mathscr{A}^{o p}, \mathscr{V}\right]\left(\mathrm{y}_{\mathscr{A}}(a), f\right)$ by Yoneda Lemma. Hence we have the desired adjunction

$$
\left(y_{\mathscr{A}}\right)_{\diamond} \dashv\left(\mathrm{y}_{\mathscr{A}}\right)^{\diamond}=\mathrm{ev}_{\mathscr{A}}
$$

proving that $(-)_{\diamond} \dashv(-)^{\dagger}$ is an adjunction of a $\mathrm{KZ}$ type.

Notation 3.9. Denote by

$$
(\mathbb{L}, y, m)
$$

the 2-monad on $\mathscr{Y}$-cat of the 2-adjunction $(-)_{\diamond} \dashv(-)^{\dagger}$.

Remark 3.10. Since the 2 -adjunction is of KZ type (i.e., an additional adjunction concerning units and counits holds), the 2 -monad $(\mathbb{L}, y, m)$ is a $K Z$ doctrine, i.e., for every category $\mathscr{A}$, there is an adjunction

$$
m_{\mathscr{A}} \dashv \mathrm{y}_{\mathrm{L} \mathscr{A}}: \mathbb{L} \mathscr{A} \longrightarrow \mathbb{L} \mathbb{L} \mathscr{A}
$$

in $\mathscr{Y}$-cat that has an isomorphism as its counit. See, e.g., [34] or [28].

Thus $\mathbb{L} \mathscr{A}$ should be thought of as a cocompletion of $\mathscr{A}$ w.r.t. a certain class of colimits. This is indeed the case: $\mathbb{L} \mathscr{A}=\left[\mathscr{A}^{o p}, \mathscr{V}\right]$ are the "presheaves on $\mathscr{A}^{\prime}, y_{\mathscr{A}}: \mathscr{A} \longrightarrow\left[\mathscr{A}^{o p}, \mathscr{V}\right]$ is the Yoneda embedding and $\mathrm{m}_{\mathscr{A}}: \mathbb{L} \mathbb{L} \mathscr{A} \longrightarrow \mathbb{L} \mathscr{A}$ is the "union mapping":

$$
\mathrm{m}_{\mathscr{A}}: W \mapsto\left(a \mapsto \bigvee_{w} W(w) \otimes w(a)\right)
$$

By general facts, see [25], ㄴ is the KZ doctrine of a free cocompletion under weighted colimits.

Remark 3.11. Above, we have again made use of the fact that we enrich in a quantale. In particular, the $\mathrm{KZ}$ doctrine $\mathbb{L}$ is a genuine 2 -functor, i.e., $\mathbb{L}$ preserves identities and composition on the nose.

This is not the case for enrichment in a general base category $\mathscr{V}$; a construction analogous to $\mathbb{L}$ is available but we end up with a mere pseudofunctor, i.e., such $\mathbb{L}$ preserves identities and composition only up to specified isomorphisms that satisfy certain coherence conditions.

The following result is now trivial since $\mathscr{V}$-modules $\mathscr{A} \longrightarrow \mathscr{B}$ correspond bijectively to Kleisli morphisms $\mathscr{A} \longrightarrow\left[\mathscr{B}^{o p}, \mathscr{V}\right]$ for the KZ doctrine $(\mathbb{L}, y, \mathrm{~m})$.

Lemma 3.12. The 2-functor $(-)^{\dagger}: \mathscr{V}$-mod $\longrightarrow \mathscr{V}$-cat exhibits $\mathscr{V}$-mod as the Kleisli 2category of the $K Z$ doctrine $(\mathbb{L}, y, m)$.

Example 3.13. In case $\mathscr{V}=2, \mathscr{V}$-mod can be identified with the category of complete join-semilattices and join-preserving maps. This follows from the fact that $(\mathbb{L}, y, m)$ is the 2-monad of complete join-semilattices on the category of preorders. 
Combining the results above, we obtain the first characterisation of functors admitting a relation lifting (Definition 3.7):

Corollary 3.14. For a 2-functor $T: \mathscr{V}$-cat $\longrightarrow \mathscr{V}$-cat, there is a one-to-one correspondence between

(1) Relation liftings $\bar{T}: \mathscr{V}$-mod $\longrightarrow \mathscr{V}$-mod and

(2) Distributive laws $\delta: T \cdot \mathbb{L} \longrightarrow \mathbb{L} \cdot T$.

Above, the distributive law of a 2-functor over a 2-monad is defined in the same way as for functors and monads. In our case, it is a 2-natural transformation $\delta: T \cdot \mathbb{L} \longrightarrow \mathbb{L} \cdot T$ making the following two diagrams
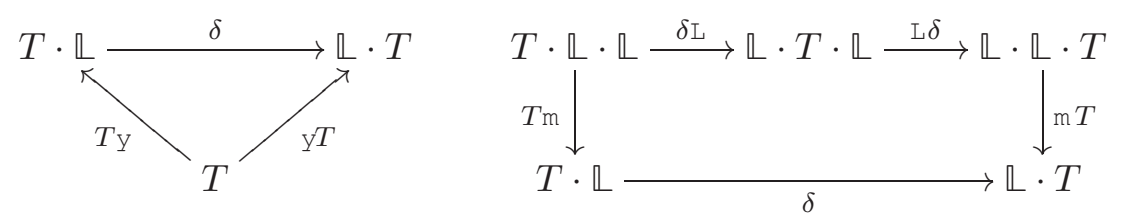

commutative. See, e.g., 34 for details.

Remark 3.15. It should be noted that, given the relation lifting $\bar{T}: \mathscr{V}$-mod $\longrightarrow \mathscr{V}$-mod of $T$, the distributive law $\delta: T \cdot \mathbb{L} \longrightarrow \mathbb{L} \cdot T$ is, at a category $\mathscr{A}$, given as follows:

To give $\delta_{\mathscr{A}}: T\left[\mathscr{A}^{o p}, \mathscr{V}\right] \longrightarrow\left[(T \mathscr{A})^{o p}, \mathscr{V}\right]$ is to give a module $R: T\left[\mathscr{A}^{o p}, \mathscr{V}\right] \longrightarrow T \mathscr{A}$ and we put $R=\bar{T}\left(\left(y_{\mathscr{A}}\right)^{\diamond}\right)$.

This process generalises the classical case, see, e.g., [29].

3.B. Modules as cospans. The idea of the second characterisation theorem for a relation lifting hinges upon a representation of modules as certain cospans in $\mathscr{Y}$-cat, called (twosided) codiscrete cofibrations. Although this notion is a bit technical, it reflects the basic perception that relations should be represented as spans that are jointly mono. This will be seen as follows:

(1) A (two-sided) codiscrete cofibration in $\mathscr{Y}$-cat becomes a (two sided) discrete fibration in $(\mathscr{H} \text {-cat })^{o p}$.

(2) The Pos-category ( $\mathscr{Y}$-cat) ${ }^{o p}$ will be proved to be "regular" w.r.t. a certain factorisation system (see Section 4 below), and the (two-sided) discrete fibrations will jointly belong to the "monomorphism part" of the factorisation system on $(\mathscr{K} \text {-cat })^{o p}$.

We will then proceed in Section 5 almost exactly as in the classical case: the relation lifting will be defined by means of graphs of relations, represented as cospans.

Definition 3.16. A collage of a module $R: \mathscr{A} \longrightarrow \mathscr{B}$ is a cospan

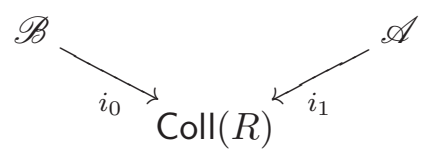

in $\mathscr{Y}$-cat, where the category $\operatorname{Coll}(R)$ is defined as follows:

(1) Objects of Coll $(R)$ are the disjoint union of objects of $\mathscr{A}$ and $\mathscr{B}$.

(2) $\operatorname{Coll}(R)\left(a, a^{\prime}\right)=\mathscr{A}\left(a, a^{\prime}\right)$ in case both $a$ and $a^{\prime}$ are in $\mathscr{A}$.

(3) Coll $(R)\left(b, b^{\prime}\right)=\mathscr{B}\left(b, b^{\prime}\right)$ in case both $b$ and $b^{\prime}$ are in $\mathscr{B}$.

(4) Coll $(R)(b, a)=R(b, a)$ in case $b$ is in $\mathscr{B}$ and $a$ is in $\mathscr{A}$. 
(5) $\operatorname{Coll}(R)(a, b)=\perp$ in case $a$ is in $\mathscr{A}$ and $b$ is in $\mathscr{B}$.

The functor $i_{0}: \mathscr{B} \longrightarrow \operatorname{Coll}(R)$ is given by the object-assignment $b \mapsto b$ and $\mathscr{B}\left(b, b^{\prime}\right)=$ $\operatorname{Coll}(R)\left(b, b^{\prime}\right)$. The functor $i_{1}: \mathscr{A} \longrightarrow \operatorname{Coll}(R)$ is defined analogously.

Remark 3.17. The collage of a module $R$ is a lax colimit of $R$ as a 1 -cell in $\mathscr{V}$-mod. More precisely, the lax triangle

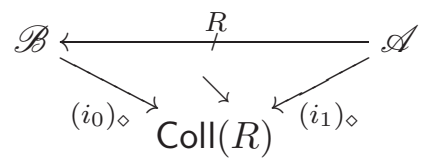

in $\mathscr{Y}$-mod, has the appropriate universal property. See [43].

Example 3.18. Suppose $f: \mathscr{A} \longrightarrow \mathscr{B}$ is a functor in $\mathscr{Y}$-cat. Then the collages of modules $f_{\diamond}$ and $f^{\diamond}$ are computed as follows:

(1) For $f_{\diamond}(b, a)=\mathscr{B}(b, f a)$, the only nontrivial hom-objects in the category $\operatorname{Coll}\left(f_{\diamond}\right)$ are

$$
\operatorname{Coll}\left(f_{\diamond}\right)(b, a)=\mathscr{B}(b, f a)
$$

(2) For $f^{\diamond}(a, b)=\mathscr{B}(f a, b)$, the only nontrivial hom-objects in the category Coll $\left(f^{\diamond}\right)$ are

$$
\operatorname{Coll}\left(f^{\diamond}\right)(a, b)=\mathscr{B}(f a, b)
$$

The proof of the following result is easy, once we employ the general definition of a two-sided discrete fibration in a general 2-category, see Remark 3.20

Lemma 3.19. Every collage $\left(i_{0}, \operatorname{Coll}(R), i_{1}\right)$ of a module $R: \mathscr{A} \longrightarrow \mathscr{B}$ is a two-sided codiscrete cofibration in $\mathscr{V}$-cat, i.e., it is a two-sided discrete fibration in ( $\mathscr{Y}$-cat) ${ }^{o p}$.

Moreover, the equality $R=\left(i_{0}\right)^{\diamond} \cdot\left(i_{1}\right)_{\diamond}$ holds in $\mathscr{V}$-mod.

Remark 3.20. Here is the "elementary" description of two-sided discrete fibrations that works in any category $\mathrm{K}$ enriched in Pre. See [45] for more details.

A span $\left(d_{0}, \mathscr{E}, d_{1}\right): \mathscr{B} \longrightarrow \mathscr{A}$ in $\mathrm{K}$ is a two-sided discrete fibration, if the following three conditions are satisfied:

(1) For each $m: \mathscr{K} \longrightarrow \mathscr{E}, a, a^{\prime}: \mathscr{K} \longrightarrow \mathscr{A}, b: \mathscr{K} \longrightarrow \mathscr{B}$ and $\alpha: a^{\prime} \longrightarrow a$ such that triangles

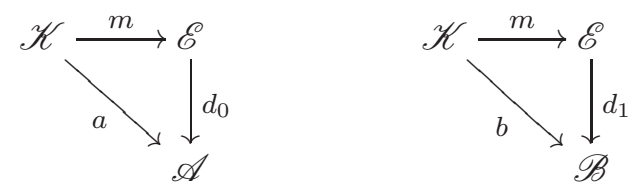

commute, there is a unique $\bar{m}: \mathscr{K} \longrightarrow \mathscr{E}$ and a unique $d_{0}^{*}(\alpha): \bar{m} \longrightarrow m$ such that
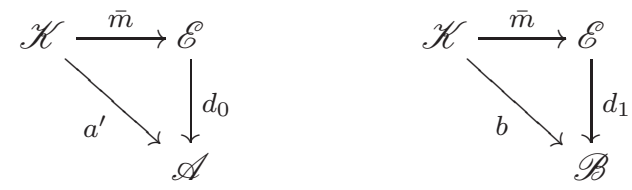

and

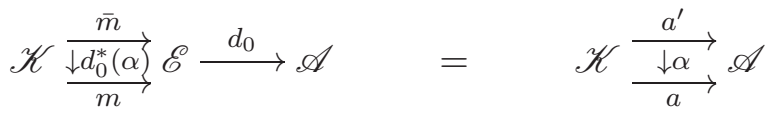

$$
\begin{aligned}
& \mathscr{K} \frac{\bar{m}}{\frac{\underline{\underline{m}}(\alpha)}{m}} \mathscr{E} \stackrel{d_{1}}{\longrightarrow} \mathscr{B}=\quad \mathscr{K} \stackrel{b}{\longrightarrow} \mathscr{B}
\end{aligned}
$$


commute. The 2-cell $d_{0}^{*}(\alpha)$ is called the cartesian lift of $\alpha$.

(2) For each $m: \mathscr{K} \longrightarrow \mathscr{E}, a: \mathscr{K} \longrightarrow \mathscr{A}, b, b^{\prime}: \mathscr{K} \longrightarrow \mathscr{B}$ and $\beta: b \longrightarrow b^{\prime}$ such that triangles
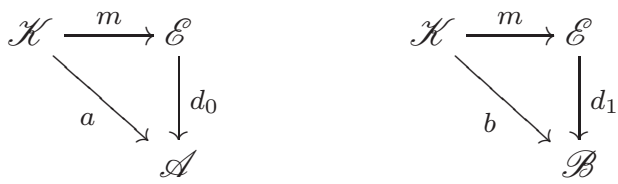

commute, there is a unique $\bar{m}: \mathscr{K} \longrightarrow \mathscr{E}$ and a unique $d_{1}^{*}(\beta): m \longrightarrow \bar{m}$ such that
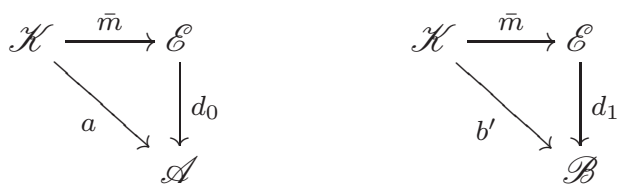

and

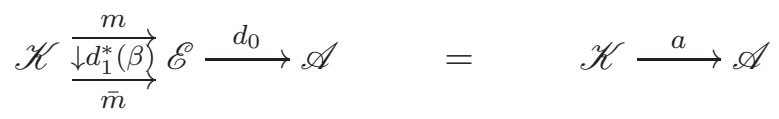

$$
\begin{aligned}
& \mathscr{K} \frac{m}{\frac{\overrightarrow{\downarrow d}(\beta)}{\bar{m}}} \mathscr{E} \stackrel{d_{1}}{\longrightarrow} \mathscr{B}=\quad \mathscr{K} \underset{\frac{\downarrow \beta}{b^{\prime}}}{\stackrel{\frac{b}{\longrightarrow}}{\longrightarrow}}
\end{aligned}
$$

commute. The 2-cell $d_{1}^{*}(\beta)$ is called the opcartesian lift of $\beta$.

(3) Given any $\sigma: m \longrightarrow m^{\prime}: K \longrightarrow E$, then the composite $d_{0}^{*}\left(d_{0} \sigma\right) \cdot d_{1}^{*}\left(d_{1} \sigma\right)$ is defined and it is equal to $\sigma$.

Remark 3.21. We indicated that every module $R$ can be represented as a two-sided codiscrete cofibration, namely the collage of $R$. Conversely, every two-sided codiscrete cofibration $\left(d_{0}, \mathscr{E}, d_{1}\right)$ in $\mathscr{V}$-cat gives rise to a module $\left(d_{0}\right)^{\diamond} \cdot\left(d_{1}\right)_{\diamond}$. That these two representations are equivalent is proved in [45, Corollary 6.16].

In what follows we will not distinguish between modules and the corresponding twosided codiscrete cofibrations.

\section{4. "Regularity" of ( $/$-cat $)^{o p}$}

In this section we introduce a factorisation system

$$
(\mathcal{E}, \mathcal{M})
$$

on the 2-category $\mathscr{Y}$-cat such that $\left(\mathscr{Y}\right.$-cat) ${ }^{o p}$ (equipped with the factorisation system $(\mathcal{M}, \mathcal{E})$ ) will become regular as a 2-category. Composing two-sided discrete fibrations in ( $\mathscr{Y}$-cat) ${ }^{o p}$ $(=$ collages in $\mathscr{Y}$-cat $)$ will then become very easy: one would take the $\mathcal{M}$-quotient of the pullback in $(\mathscr{Y} \text {-cat })^{o p}$ of two spans exactly as in sets. Since this will happen in $(\mathscr{K} \text {-cat })^{o p}$, we will, in reality, take a subobject of a pushout of two collages.

In fact, our approach to codiscrete cofibrations and their composition then mimics the way how relations are treated and composed in an ordinary (i.e., non-enriched) regular category, see, e.g., [15].

The factorisation system is going to be introduced in the following manner:

(1) We choose a class $\mathcal{C}$ that will consist of functors in $\mathscr{Y}$-cat of the form $\left[i_{0}, i_{1}\right]: \mathscr{B}+\mathscr{A} \longrightarrow$ Coll $(R)$, where $\left(i_{0}, \operatorname{Coll}(R), i_{1}\right)$ is the collage of a module $R: \mathscr{A} \longrightarrow \mathscr{B}$. 
(2) We identify the class $\mathcal{M}=\mathcal{C}^{\downarrow}$ of functors in $\mathscr{V}$-cat that are orthogonal to all functors in $\mathcal{C}$ as exactly the fully faithful functors.

(3) By general facts about factorisation systems, if we put $\mathcal{E}=\mathcal{M}^{\uparrow}$ to be the class of functors orthogonal to $\mathcal{M}$, then $(\mathcal{E}, \mathcal{M})$ is a factorisation system in $\mathscr{Y}$-cat.

The so defined factorisation system $(\mathcal{E}, \mathcal{M})$ will turn $\mathscr{Y}$-cat into a "coregular 2-category": the class $\mathcal{M}$ will be proved to be stable under pushouts and tensoring with the poset 2 . The proof of $(\mathcal{E}, \mathcal{M})$-coregularity of $\mathscr{V}$-cat can also be found in [37], we include the proofs here for the sake of self-containedness. Although the notion of "(co)regularity" for general 2 -categories is somewhat intricate, see [44, 14, we will benefit again from the fact that $\mathscr{Y}$-cat is enriched in preorders.

Definition 4.1. Say that $e: \mathscr{A} \longrightarrow \mathscr{B}$ is orthogonal to $j: \mathscr{C} \longrightarrow \mathscr{D}$, provided that the following square

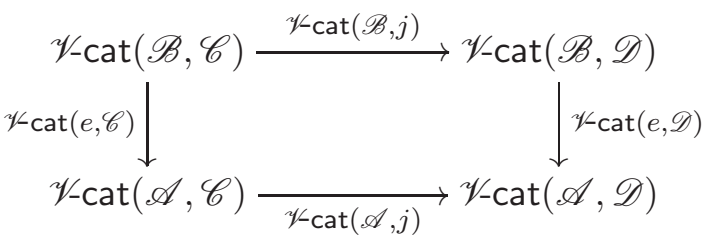

is a pullback in the category of preorders.

Remark 4.2. It should help to think of $e$ as being "epi" and $j$ as being "mono". The above pullback then states the unique diagonalisation property w.r.t. both 1-cells and 2-cells.

We will freely adopt the usual notation and terminology: given a class $\mathcal{C}$ of "epis", we denote by $\mathcal{C} \downarrow$ the class of "monos" orthogonal to every element of $\mathcal{C}$. Dually, we denote by $\mathcal{C}^{\uparrow}$ the class of all "epis", orthogonal to every "mono" in $\mathcal{C}$.

Definition 4.3. A functor $f: \mathscr{A} \longrightarrow \mathscr{B}$ is called fully faithful if we have the equality $\mathscr{A}\left(a, a^{\prime}\right)=\mathscr{B}\left(f a, f a^{\prime}\right)$ for all $a, a^{\prime}$ in $\mathscr{A}$.

Example 4.4. In case $\mathscr{V}=2$, the fully faithful functors are exactly the order-embeddings. In case $\mathscr{V}=[0 ; 1]$, the fully faithful functors are exactly the isometries.

Lemma 4.5. For a functor $f: \mathscr{A} \longrightarrow \mathscr{B}$, the following are equivalent:

(1) $f$ is fully faithful.

(2) The unit $\eta^{f}$ of $f_{\diamond} \dashv f^{\diamond}$ is an isomorphism.

Moreover, the conditions above imply

$f$ is representably fully faithful, i.e., the monotone map

$$
\mathscr{V} \text {-cat }(\mathscr{X}, f): \mathscr{V} \text {-cat }(\mathscr{X}, \mathscr{A}) \longrightarrow \mathscr{V} \text {-cat }(\mathscr{X}, \mathscr{B})
$$

is fully faithful (= reflects preorders), for every $\mathscr{X}$.

Proof. The equivalence of the first two items is obvious: the unit $\eta^{f}$ is manifested by the inequality $\mathscr{A}\left(a, a^{\prime}\right) \leq \mathscr{B}\left(f a, f a^{\prime}\right)=\bigvee_{b} \mathscr{B}\left(b, f a^{\prime}\right) \otimes \mathscr{B}(f a, b)$.

To prove the implication, consider a pair $h, k: \mathscr{X} \longrightarrow \mathscr{A}$. Then

$$
\mathscr{Y}-\operatorname{cat}(\mathscr{X}, \mathscr{A})(h, k)=\bigwedge_{x} \mathscr{A}(h x, k x)=\bigwedge_{x} \mathscr{B}(f h x, f k x)=\mathscr{V}-\operatorname{cat}(\mathscr{X}, \mathscr{B})(f h, f k)
$$

proves that $f$ is representably fully faithful.

Note that the converse would only hold if we considered $\mathscr{V}$-cat to be a $\mathscr{V}$-category rather than a Pre-category. 
Lemma 4.6. Define $\mathcal{C}$ to be the class of functors in $\mathscr{Y}$-cat of the form $\left[i_{0}, i_{1}\right]: \mathscr{B}+\mathscr{A} \longrightarrow$ Coll $(R)$, where $\left(i_{0}\right.$, Coll $\left.(R), i_{1}\right)$ is the collage of a module $R: \mathscr{A} \longrightarrow \mathscr{B}$. Define $\mathcal{M}$ to be the class of all functors orthogonal to $\mathcal{C}$, i.e., put $\mathcal{M}=\mathcal{C}^{\downarrow}$. Then $\mathcal{M}$ consists exactly of the fully faithful functors.

Proof. Suppose $j: \mathscr{C} \longrightarrow \mathscr{D}$ is a fully faithful functor. Consider $\left[i_{0}, i_{1}\right]: \mathscr{B}+\mathscr{A} \longrightarrow \operatorname{Coll}(R)$ in $\mathcal{C}$. Then $\left[i_{0}, i_{1}\right]$ is bijective on objects. To prove the existence of unique diagonalisation, that is, to prove that

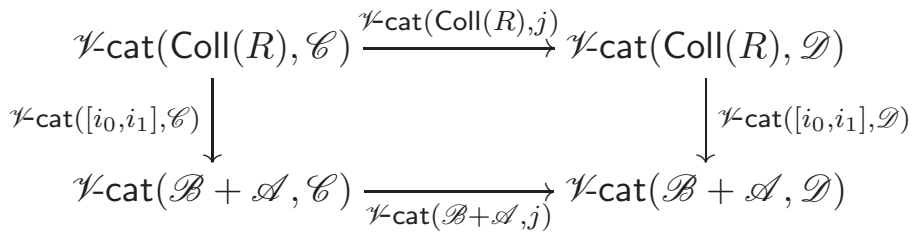

is a pullback in Pre, consider any pair $u: \mathscr{B}+\mathscr{A} \longrightarrow \mathscr{C}, v: \operatorname{Coll}(R) \longrightarrow \mathscr{D}$ such that

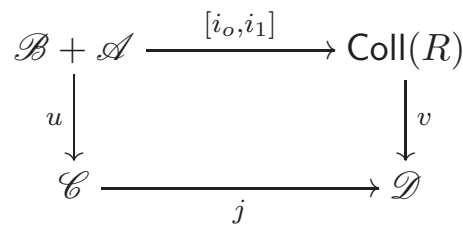

commutes in $\mathscr{Y}$-cat. Since $\left[i_{0}, i_{1}\right]$ is bijective on objects, one can define an object assignment of a functor $d: \operatorname{Coll}(R) \longrightarrow \mathscr{C}$ as the object assignment of $u$. Since $j$ is fully faithful, $d$ so defined will be a functor.

Conversely, suppose $f: \mathscr{A} \longrightarrow \mathscr{B}$ is orthogonal to every functor in $\mathcal{C}$. Consider any $a$, $a^{\prime}$ in $\mathscr{A}$ and define a module $R$ from $\mathscr{I}$ to $\mathscr{I}$ (where $\mathscr{I}$ is the unit category on one object $*$ with $\mathscr{I}(*, *)=I)$ with $R(*, *)=\mathscr{B}\left(f a, f a^{\prime}\right)$. Then the square

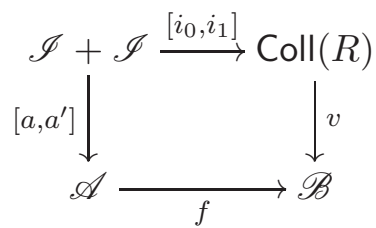

commutes, where $v: \operatorname{Coll}(R) \longrightarrow \mathscr{B}$ is given by $b \mapsto b$ for $b$ in $\mathscr{B}, x \mapsto f x$ for $x$ in $\mathscr{A}$.

Hence there is a unique diagonal $d: \operatorname{Coll}(R) \longrightarrow \mathscr{A}$, witnessing the inequality $\mathscr{B}\left(f a, f a^{\prime}\right) \leq$ $\mathscr{A}\left(a, a^{\prime}\right)$. Hence $\mathscr{A}\left(a, a^{\prime}\right)=\mathscr{B}\left(f a, f a^{\prime}\right)$ which we were supposed to prove.

Remark 4.7. Functors that are orthogonal to every fully faithful functor in $\mathscr{V}$-cat are called eso functors (where eso stands for essentially surjective on objects).

Their defining property is the following one: $e: \mathscr{A} \longrightarrow \mathscr{B}$ is an eso functor in $\mathscr{Y}$-cat iff the following diagram

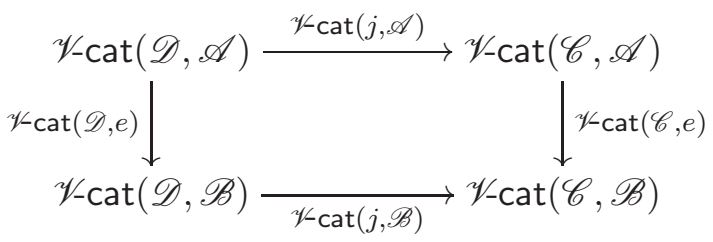

is a pullback in Pre, for every fully faithful $j: \mathscr{C} \longrightarrow \mathscr{D}$. 
Observe that any functor $e: \mathscr{A} \longrightarrow \mathscr{B}$ in $\mathscr{V}$-cat with the property that $a \mapsto e a$ is a bijection is an eso functor.

By putting $\mathcal{E}=\mathcal{M}^{\uparrow}$ we define a factorisation system on $\mathscr{V}$-cat as long as we know that factorisations exist. Their existence is established easily.

Lemma 4.8. Any functor $f: \mathscr{A} \longrightarrow \mathscr{B}$ can be written as a composite $j \cdot e$ where $j$ is fully faithful and e surjective on objects.

Proof. Define $\mathscr{C}$ to have the same objects as $\mathscr{A}$ and put $\mathscr{C}\left(a, a^{\prime}\right)=\mathscr{B}\left(f a, f a^{\prime}\right)$. Clearly, $\mathscr{C}$ is a category: $I \leq \mathscr{C}(a, a)=\mathscr{B}(f a, f a)$ holds, since $f$ is a functor and $\mathscr{C}\left(a, a^{\prime}\right) \otimes \mathscr{C}\left(a^{\prime}, a^{\prime \prime}\right) \leq$ $\mathscr{C}\left(a, a^{\prime \prime}\right)$ is the inequality $\mathscr{B}\left(f a, f a^{\prime}\right) \otimes \mathscr{B}\left(f a^{\prime}, f a^{\prime \prime}\right) \leq \mathscr{B}\left(f a, f a^{\prime \prime}\right)$ that holds since $f$ is a functor.

Define $j: \mathscr{C} \longrightarrow \mathscr{B}$ by the object-assignment $a \mapsto f a$ and $\mathscr{C}\left(a, a^{\prime}\right)=\mathscr{B}(f a, f a)$. Then $j$ is fully faithful.

Define $e: \mathscr{A} \longrightarrow \mathscr{C}$ by the object-assignment $a \mapsto a$ and $\mathscr{A}\left(a, a^{\prime}\right) \leq \mathscr{C}\left(a, a^{\prime}\right)=$ $\mathscr{B}\left(f a, f a^{\prime}\right)$ (which holds since $f$ is a functor).

Clearly: $f=j \cdot e$.

The next two results conclude "coregularity" of our factorisation system.

Lemma 4.9. The class of fully faithful functors is stable under pushouts.

Proof. Suppose that

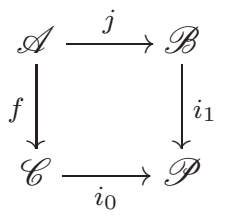

is a pushout in $\mathscr{Y}$-cat and suppose $j$ is fully faithful. We need to prove that $i_{0}$ is fully faithful.

Recall from 1.15 in 14 that the objects of $\mathscr{P}$ are a disjoint union of the objects of $\mathscr{C}$ and of those objects of $\mathscr{B}$ that are not in $\mathscr{A}$. The hom-objects are defined as follows:

$$
\begin{aligned}
\mathscr{P}(c, b) & =\bigvee_{a} \mathscr{C}(c, f a) \otimes \mathscr{B}(j a, b) \\
\mathscr{P}(b, c) & =\bigvee_{a} \mathscr{B}(b, j a) \otimes \mathscr{C}(f a, c) \\
\mathscr{P}\left(b, b^{\prime}\right) & =\bigvee_{a, a^{\prime}} \mathscr{B}(b, j a) \otimes \mathscr{C}\left(f a, f a^{\prime}\right) \otimes \mathscr{B}\left(j a^{\prime}, b^{\prime}\right) \\
\mathscr{P}\left(c, c^{\prime}\right) & =\mathscr{C}\left(c, c^{\prime}\right)
\end{aligned}
$$

where $b, b^{\prime}$ are in $\mathscr{B}$ but not in $\mathscr{A}$, and $c, c^{\prime}$ are in $\mathscr{C}$. The composition and identities in $\mathscr{P}$ are given by those of $\mathscr{C}$ and $\mathscr{B}$.

The functor $i_{0}$ is given by $c \mapsto c$, the functor $i_{1}$ by $b \mapsto b$ if $b$ is not in $\mathscr{A}$ and by $a \mapsto f a$ otherwise.

Clearly, $i_{0}$ is fully faithful. 
Recall that 2 is the two-element chain, i.e., a preorder. Recall also that $\mathscr{Y}$-cat is a 2 category and, in fact, $\mathscr{Y}$-cat is enriched in preorders. Thus it makes a perfect sense to define a tensor by 2 for any small category $\mathscr{A}$. This amounts to give a category $2 * \mathscr{A}$ together with an isomorphism

natural in $\mathscr{B}$.

$$
\mathscr{Y}-\operatorname{cat}(2 * \mathscr{A}, \mathscr{B}) \cong \operatorname{Pre}(2,[\mathscr{A}, \mathscr{B}])
$$

An explicit description of $2 * \mathscr{A}$ is as follows:

(1) Objects are pairs $(0, a),(1, a)$, where $a$ is in $\mathscr{A}$.

(2) The hom-objects are defined in the following manner:

$$
\begin{aligned}
& 2 * \mathscr{A}\left((0, a),\left(0, a^{\prime}\right)\right)=\mathscr{A}\left(a, a^{\prime}\right) \\
& 2 * \mathscr{A}\left((1, a),\left(1, a^{\prime}\right)\right)=\mathscr{A}\left(a, a^{\prime}\right) \\
& 2 * \mathscr{A}\left((0, a),\left(1, a^{\prime}\right)\right)=I \\
& 2 * \mathscr{A}\left((1, a),\left(0, a^{\prime}\right)\right)=\perp
\end{aligned}
$$

Hence to give a functor $f: 2 * \mathscr{A} \longrightarrow \mathscr{B}$ amounts to giving a pair $f_{0}: \mathscr{A} \longrightarrow \mathscr{B}$, $f_{1}: \mathscr{A} \longrightarrow \mathscr{B}$ of functors with $f_{0} \leq f_{1}$.

Lemma 4.10. Fully faithful functors are closed under tensoring with 2.

Proof. Suppose $j: \mathscr{A} \longrightarrow \mathscr{B}$ is fully faithful and consider $2 * j: 2 * \mathscr{A} \longrightarrow 2 * \mathscr{B}$. That $j$ is fully faithful follows immediately from the description of tensoring with 2 .

\section{The RELATiOn Lifting Via COSPANS}

The condition on $T: \mathscr{Y}$-cat $\longrightarrow \mathscr{Y}$-cat to admit a relation lifting $\bar{T}: \mathscr{V}$-mod $\longrightarrow \mathscr{Y}$-mod using cospans is related to two facts:

(1) Collages compose by taking $\mathcal{M}$-subobjects of pushouts.

(2) One can define an assignment $\bar{T}$ on arrows of $\mathscr{Y}$-mod and prove that $\bar{T}$ respects composition of collages, if $T$ "behaves nicely" w.r.t. certain lax commutative squares. This is Proposition 5.7 below.

In fact, as we will see in Theorem 5.10 below, the graph 2 -functor $(-)_{\diamond}: \mathscr{Y}$-cat $\longrightarrow \mathscr{V}$-mod enjoys a certain universal property that will allow us to isolate the necessary and sufficient condition on $T$ to admit a relation lifting. This is our main result, see Corollary 5.11 below. The nature of the universal property of the graph 2-functor goes back to [21].

The composition of collages. The composition of collages is performed in the same way as in any coregular category. Namely, given two-sided codiscrete cofibrations
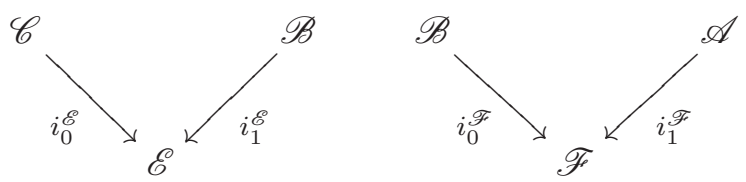
in $\mathscr{V}$-cat, form the diagram

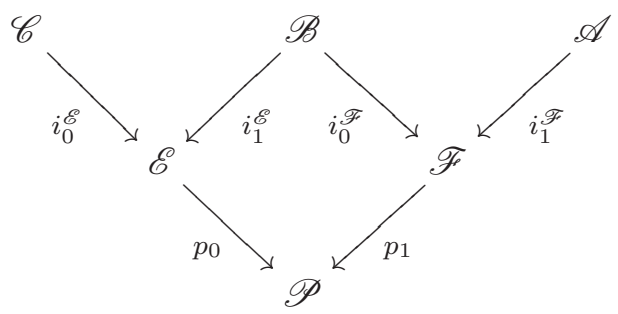

where the middle square is a pushout, then form the functor

$$
\left[p_{0} i_{0}^{\mathscr{E}}, p_{1} i_{1}^{\mathscr{F}}\right]: \mathscr{C}+\mathscr{A} \longrightarrow \mathscr{P}
$$

and consider its $(\mathcal{E}, \mathcal{M})$-factorisation

$$
\mathscr{C}+\mathscr{A} \stackrel{\left[i_{0}^{\mathscr{E} O \mathscr{F}}, i_{1}^{\mathscr{E} \circ \mathscr{F}}\right]}{\longrightarrow} \mathscr{E} \circ \mathscr{F} \stackrel{j}{\longrightarrow} \mathscr{P}
$$

Then the cospan

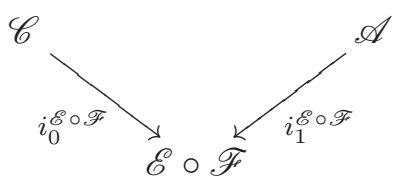

is the composite of codiscrete cofibrations $\mathscr{E}$ and $\mathscr{F}$. This follows immediately from [14, Theorem 4.20]: our factorisation system $(\mathscr{E}, \mathscr{M})$ on $\mathscr{Y}$-cat satisfies all the assumptions of this theorem.

The idea of a relation lifting. Given a collage $\left(i_{0}\right.$, Coll $\left.(R), i_{1}\right)$ of a module $R$ from $\mathscr{A}$ to $\mathscr{B}$, we want to define

$$
\bar{T}(R) \equiv T \mathscr{A} \stackrel{\left(T i_{1}\right)_{\diamond}}{\longrightarrow} T \operatorname{Coll}(R) \stackrel{\left(T i_{0}\right)^{\diamond}}{\longrightarrow} T \mathscr{B}
$$

as the value of the relation lifting. We now indicate how this definition yields a functor, provided $T$ satisfies an additional condition. Namely, we will want $T: \mathscr{V}$-cat $\longrightarrow \mathscr{Y}$-cat to preserve a certain property that is called exactness of a lax square, see [19].

Definition 5.1. Call a lax square

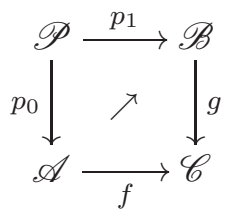

in $\mathscr{Y}$-cat exact, if the equality

$$
\mathscr{C}(f a, g b)=\bigvee_{w} \mathscr{A}\left(a, p_{0} w\right) \otimes \mathscr{B}\left(p_{1} w, b\right)
$$

holds, naturally in $a$ and $b$. 
Remark 5.2. The exactness condition (5.1) above is clearly equivalent to the equality

$$
f^{\diamond} \cdot g_{\diamond}=\left(p_{0}\right)_{\diamond} \cdot\left(p_{1}\right)^{\diamond}
$$

in $\mathscr{V}$-mod. Let us further remark that every lax square is "nearly exact" in the sense that the inequality

$$
\mathscr{C}(f a, g b) \geq \bigvee_{w} \mathscr{A}\left(a, p_{0} w\right) \otimes \mathscr{B}\left(p_{1} w, b\right)
$$

holds, for all $a$ and $b$. Indeed, consider the following inequalities

$$
\begin{aligned}
\bigvee_{w} \mathscr{A}\left(a, p_{0} w\right) \otimes \mathscr{B}\left(p_{1} w, b\right) & \leq \bigvee_{w} \mathscr{C}\left(f a, f p_{0} w\right) \otimes \mathscr{C}\left(g p_{1} w, g b\right) \\
& \leq \bigvee_{w} \mathscr{C}\left(f a, g p_{1} w\right) \otimes \mathscr{C}\left(g p_{1} w, g b\right) \\
& \leq \mathscr{C}(f a, g b)
\end{aligned}
$$

where we have used (in that order) that $f$ and $g$ are functors, that the square is lax, and the composition inequality in $\mathscr{C}$.

Example 5.3. We give examples of exact squares in $\mathscr{Y}$-cat. They all come from Guitart's paper [19], Example 1.14. The reasoning behind what follows is the exactness supremum formula (15.1) above.

(1) The squares

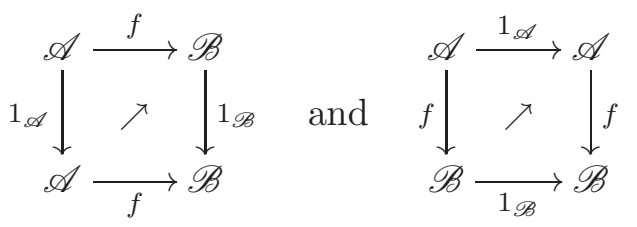

where the comparisons are identities, are always exact: the equalities

$$
\mathscr{B}(f a, b)=\bigvee_{w} \mathscr{A}(a, w) \otimes \mathscr{B}(f w, b) \quad \text { and } \quad \mathscr{B}(b, f a)=\bigvee_{w} \mathscr{B}(b, f w) \otimes \mathscr{A}(w, a)
$$

hold by the Yoneda Lemma. Such squares are called Yoneda squares in [19].

(2) Every comma square

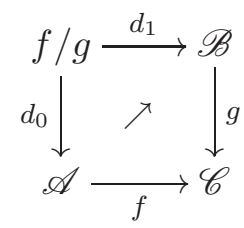

and every cocomma square

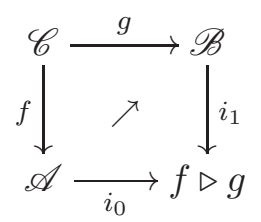

is exact. See [26] for the exact properties of comma and cocomma objects. 
(3) The square

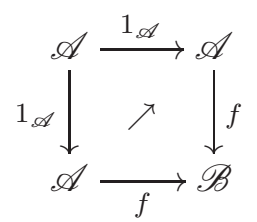

(where the comparison is identity) is exact iff $f$ is fully faithful.

(4) The square

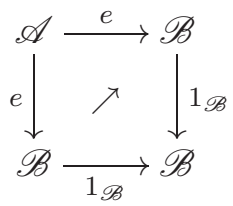

(where the comparison is identity) is exact iff $e$ is absolutely dense, i.e., iff the equality

$$
\mathscr{B}\left(b, b^{\prime}\right)=\bigvee_{a} \mathscr{B}(b, e a) \otimes \mathscr{B}\left(e a, b^{\prime}\right)
$$

holds, naturally in $b$ and $b^{\prime}$. See, e.g., 3] and 8, for more details on absolutely dense functors.

(5) The square

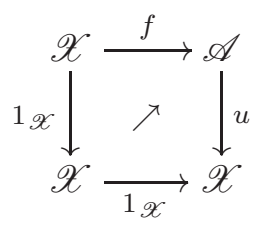

is exact iff $f \dashv u: \mathscr{A} \longrightarrow \mathscr{X}$ holds. Moreover, the comparison in the above square is the unit of $f \dashv u$.

(6) The square

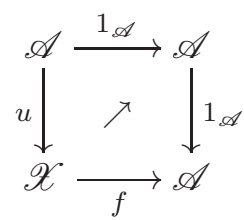

is exact iff $f \dashv u: \mathscr{A} \longrightarrow \mathscr{X}$ holds. Moreover, the comparison in the above square is the counit of $f \dashv u$.

(7) The square

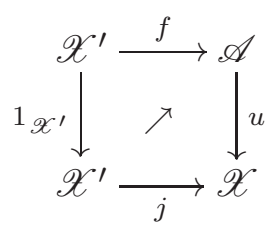

is exact iff $f \dashv_{j} u: \mathscr{A} \longrightarrow \mathscr{X}$ holds, i.e., iff $f$ is a left adjoint of $u$ relative to $j$.

Relative adjointness means the existence of an equality

$$
\mathscr{X}\left(j x^{\prime}, u a\right)=\mathscr{A}\left(f x^{\prime}, a\right)
$$


natural in $x^{\prime}$ and $a$, and due to the equality

$$
\mathscr{A}\left(f x^{\prime}, a\right)=\bigvee_{w} \mathscr{X}^{\prime}\left(w, x^{\prime}\right) \otimes \mathscr{A}(f w, a)
$$

this means precisely the exactness of the above square.

(8) The square

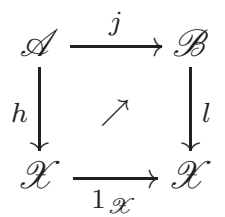

is exact iff the comparison exhibits $l$ as an absolute left Kan extension of $h$ along $j$. In fact, the equality

$$
\mathscr{X}(x, l b)=\bigvee_{a} \mathscr{X}(x, h a) \otimes \mathscr{B}(j a, b)
$$

natural in $x$ and $b$ asserts precisely the following two conditions:

(1) $l$ is a left Kan extension of $h$ along $j$.

For any $k: \mathscr{B} \longrightarrow \mathscr{X}$ we need to prove $l \leq k$ iff $h \leq k \cdot j$.

(1) Suppose $I \leq \bigwedge_{b} \mathscr{X}(l b, k b)$. Choose any $a$. Then $I \leq \mathscr{X}(h a, l j a)$ by the square above. Since $I \leq \mathscr{X}(\operatorname{lj} a, k j a)$ by assumption, $I \leq \mathscr{X}(h a, k j a)$ follows. Hence $I \leq \bigwedge_{a} \mathscr{X}(h a, k j a)$ holds.

(2) Suppose $I \leq \bigwedge_{a} \mathscr{X}(h a, k j a)$. To prove $I \leq \bigwedge_{b} \mathscr{X}(l b, k b)$, it suffices to prove that $I \leq \mathscr{X}(x, l b)$ implies $I \leq \mathscr{X}(x, k b)$, for all $x$ and $b$. Suppose $I \leq \mathscr{X}(x, l b)$. Then $I \leq \bigvee_{a} \mathscr{X}(x, h a) \otimes \mathscr{B}(j a, b)$ by the exactness formula. But

$$
\bigvee_{a} \mathscr{X}(x, h a) \otimes \mathscr{B}(j a, b) \leq \bigvee_{a} \mathscr{X}(x, h a) \otimes \mathscr{X}(k j a, k b) \leq \mathscr{X}(x, k b)
$$

since $k$ is a functor and since $h \leq k \cdot j$ holds. Thus $I \leq \mathscr{X}(x, k b)$.

(2) $l$ is an absolute left Kan extension of $h$ along $j$.

We need to prove that for any $f: \mathscr{X} \longrightarrow \mathscr{X}^{\prime}, f \cdot l$ is a left Kan extension of $f \cdot h$ along $j$. That is, for any $k: \mathscr{B} \longrightarrow \mathscr{X}^{\prime}$ we need to prove $f \cdot l \leq k$ iff $f \cdot h \leq k \cdot j$. This is proved in the same manner as above.

Observe that item (5) above is a special case of absolute Kan extensions by Bénabou's Formal Adjoint Functor Theorem [9]: $f \dashv u$ holds iff the unit exhibits $u$ as an absolute left Kan extension of identity along $f$.

Example 5.4. If the square on the left is exact, then so is the square on the right:
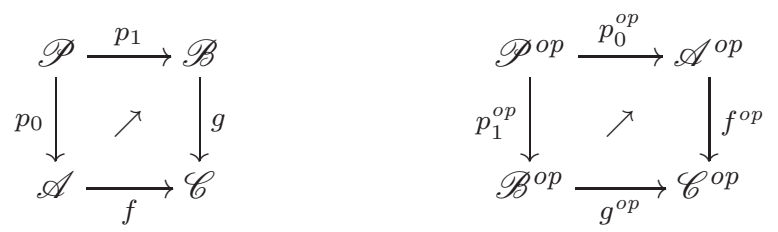
Example 5.5. Suppose that in a lax square

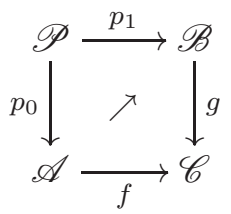

both $f$ and $p_{1}$ are left adjoints, where we denote by $f^{r}$ and $p_{1}^{r}$ the respective right adjoints.

Then the above square is exact iff there is an isomorphism $p_{0} \cdot p_{1}^{r} \cong f^{r} \cdot g$.

We will need the following technical result concerning the behaviour of the composition of collages 1

Proposition 5.6. Codiscrete cofibrations may be alternatively composed by forming factorisations of cocomma objects. More precisely, consider modules $R$ and $S$, and form the following diagram:

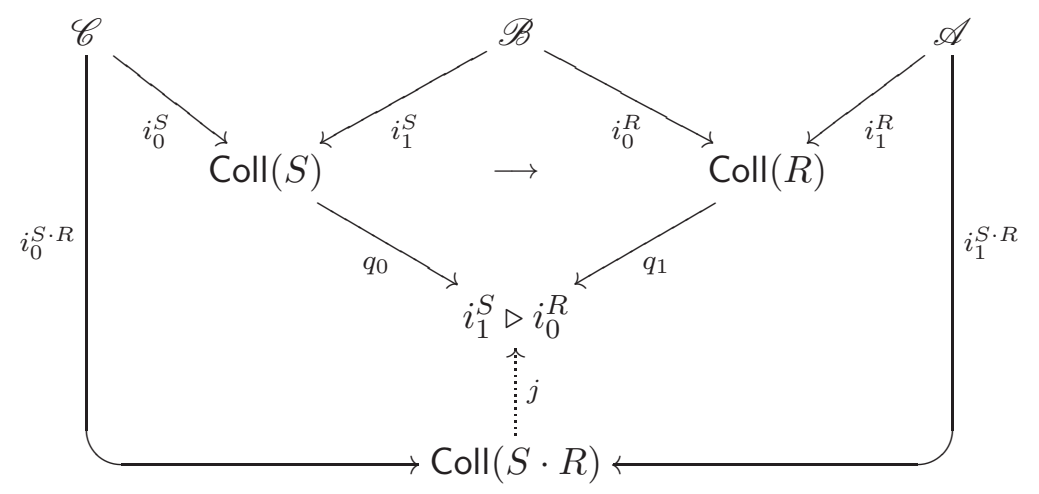

Then there exists a fully faithful $j: \operatorname{Coll}(S \cdot R) \longrightarrow i_{1}^{S} \triangleright i_{0}^{R}$ making both triangles commutative.

Proof. Observe that the category $i_{1}^{S} \triangleright i_{0}^{R}$ consists of Coll $(S)$ and Coll $(R)$ "glued" together by $\mathscr{B}$.

More precisely, the objects of $\left(i_{1}^{S} \triangleright i_{0}^{R}\right)$ are precisely those of the form $i_{0}^{S} c, i_{1}^{S} b, i_{0}^{R} b$ and $i_{1}^{R} a$. The nontrivial hom-objects are defined as follows:

$$
\begin{aligned}
\left(i_{1}^{S} \triangleright i_{0}^{R}\right)\left(i_{0}^{S} c, i_{1}^{S} b\right) & =S(c, b) \\
\left(i_{1}^{S} \triangleright i_{0}^{R}\right)\left(i_{0}^{R} b, i_{1}^{R} a\right) & =R(b, a) \\
\left(i_{1}^{S} \triangleright i_{0}^{R}\right)\left(i_{1}^{S} b, i_{0}^{R} b^{\prime}\right) & =\mathscr{B}\left(b, b^{\prime}\right) \\
\left(i_{1}^{S} \triangleright i_{0}^{R}\right)\left(i_{0}^{S} c, i_{1}^{R} a\right) & =\bigvee_{b} S(c, b) \otimes R(b, a)
\end{aligned}
$$

Hom-objects for all other combinations are defined in the obvious way.

Then $j: \operatorname{Coll}(S \cdot R) \longrightarrow i_{1}^{S} \triangleright i_{0}^{R}$ is defined by

$$
j a=i_{1}^{R} a, \quad j c=i_{0}^{S} c
$$

on objects and the equality

$$
\left(i_{1}^{S} \triangleright i_{0}^{R}\right)(j c, j a)=\bigvee_{b} \operatorname{Coll}(S)(c, b) \otimes \operatorname{Coll}(R)(b, a)
$$

\footnotetext{
${ }^{1}$ We are grateful to the anonymous referee for suggesting this.
} 
proves that $j$ is fully faithful.

We are now ready to formulate and prove the sufficiency condition of our relation lifting result.

Proposition 5.7. Suppose $T: \mathscr{V}$-cat $\longrightarrow \mathscr{V}$-cat preserves exact squares. Define the assignment $\bar{T}: \mathscr{A} \mapsto T \mathscr{A}$ on objects of $\mathscr{V}$-mod and the assignment $R \mapsto \bar{T}(R)$ by putting

$$
\bar{T}(R)=\left(T i_{0}\right)^{\diamond} \cdot\left(T i_{1}\right)_{\diamond}
$$

where $\left(i_{0}, \operatorname{Coll}(R), i_{1}\right)$ is the collage of the module $R$.

If $T$ preserves exact squares, the above assignment extends to a 2 -functor $\bar{T}: \mathscr{V}$-mod $\longrightarrow$ $\mathscr{V}$-mod such that the square

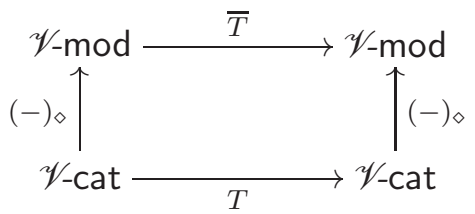

commutes.

Proof. We first prove that $\bar{T}$ preserves identities and composition.

(1) Preservation of identities.

The collage of the identity module $i d_{\mathscr{A}}$ on $\mathscr{A}$ is given by a cospan

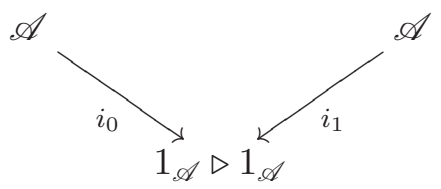

coming from the cocomma object

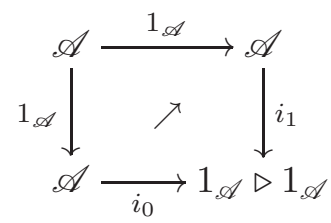

in $\mathscr{V}$-cat.

Since cocomma squares are always exact, we have that

$$
\bar{T}\left(i d_{\mathscr{A}}\right)=\left(T i_{0}\right)^{\diamond} \cdot\left(T i_{1}\right)_{\diamond}=\left(T 1_{\mathscr{A}}\right)_{\diamond} \cdot\left(T 1_{\mathscr{A}}\right)^{\diamond}=1_{T \mathscr{A}}
$$

provided that $T$ preserves the exact cocomma square above.

(2) Preservation of composition. 
We have a diagram

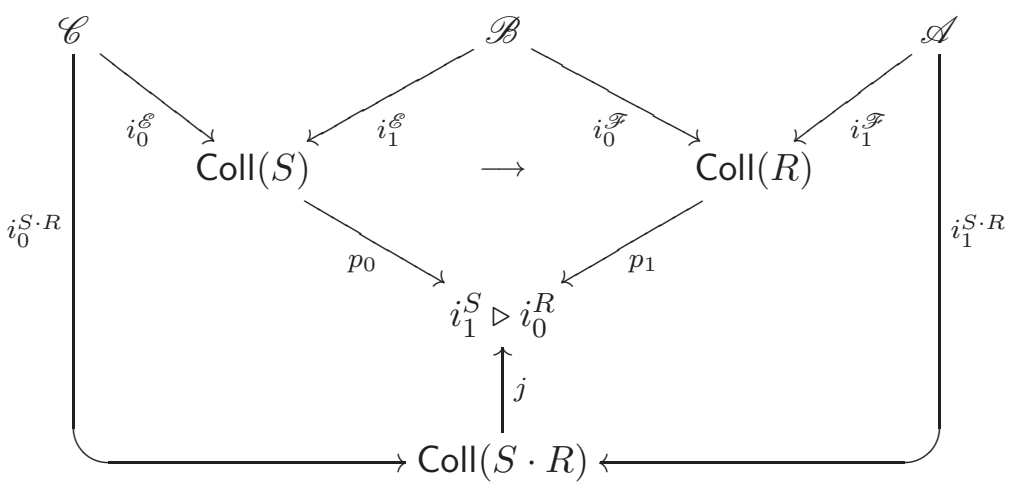

where the middle square is a cocomma square, hence exact.

By Proposition 5.6 above, there exists a fully faithful $j$. Thus $j^{\diamond} \cdot j_{\diamond}=1$ holds in $\mathscr{V}$-mod by Lemma 4.5. Therefore if we assume that $T$ preserves both the exact cocomma square above and fully faithful functors, we are done in proving that $\bar{T}$ preserves composition.

But, in general, $j: \mathscr{A} \longrightarrow \mathscr{B}$ being fully faithful is expressible by an exact square, see $5.3(3)$.

Hence if $T$ preserves exact squares, $\bar{T}$ preserves the composition. The proof is quite easy: the exactness conditions allow us to "cancel out" the mediating morphisms in $\mathscr{Y}$-mod. This is analogous to that in [10] and the idea goes back to [21].

To prove that $\bar{T}$ indeed extends $T$, observe that this is certainly so on objects. To prove that the equality

$$
(T f)_{\diamond}=\bar{T}\left(f_{\diamond}\right)
$$

holds for any $f: \mathscr{A} \longrightarrow \mathscr{B}$, it suffices to observe the following: the collage of $f_{\diamond}$ is given by the cospan of the cocomma square

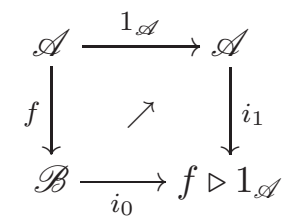

Since every cocomma square is exact by Example 5.3.(2), the lax square

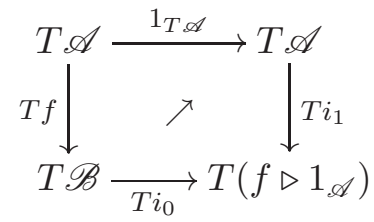

is exact by the assumption on $T$. Hence we proved the desired equality

$$
\bar{T}\left(f_{\diamond}\right)=\left(T i_{0}\right)^{\diamond} \cdot\left(T i_{1}\right)_{\diamond}=(T f)_{\diamond} \cdot\left(1_{T \mathscr{A}}\right)^{\diamond}=(T f)_{\diamond}
$$

Above, the middle equality expresses the exactness of the lax square (5.4). 
Remark 5.8. Our original proof of Proposition 5.7 used a pushout diagram in lieu of the cocomma square in diagram (5.3), so that this modified diagram represents composition of codiscrete cofibrations. The proof with a pushout square goes through unchanged, after one proves that the pushout is an exact square. The referee's suggestion to look at cocomma squares instead of pushouts yields a shorter argument (after one proves Proposition 5.6).

Definition 5.9. We say that $T: \mathscr{Y}$-cat $\longrightarrow \mathscr{Y}$-cat satisfies the Beck-Chevalley Condition $(B C C$, for short), provided it preserves exact squares.

We prove now that the satisfaction of $\mathrm{BCC}$ is equivalent to the existence of a relation lifting. To that end, we prove that the graph 2 -functor $(-)_{\diamond}$ from $\mathscr{V}$-cat to $\mathscr{Y}$-mod has essentially the same universal property as it does in the case of preorders in [10]. We only need to trade absolutely dense for fully faithful.

Theorem 5.10. Given a commutative quantale $\mathscr{V}$, the 2-functor $(-)_{\diamond}: \mathscr{V}$-cat $\longrightarrow \mathscr{V}$-mod is universal w.r.t. the following four properties:

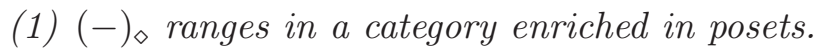

(2) Every $f_{\diamond}$ is a left adjoint (its right adjoint being denoted by $f^{\diamond}$ ).

(3) For every exact square

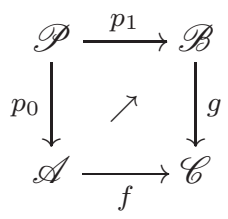

in $\mathscr{V}$-cat, we have the equality

$$
\left(p_{0}\right)_{\diamond} \cdot\left(p_{1}\right)^{\diamond}=(f)^{\diamond} \cdot(g)_{\diamond}
$$

in $\mathscr{V}$-mod.

(4) For every fully faithful $j$ in $\mathscr{V}$-cat we have $j^{\diamond} \cdot j_{\diamond}=1$ in $\mathscr{V}$-mod.

Proof. Clearly, the 2-functor $(-)_{\diamond}$ has the listed four properties.

Suppose that $F: \mathscr{V}$-cat $\longrightarrow \mathrm{K}$ is a 2 -functor having the four properties above. We want to define a unique 2-functor $F^{\sharp}: \mathscr{V}$-mod $\longrightarrow \mathrm{K}$ such that the triangle

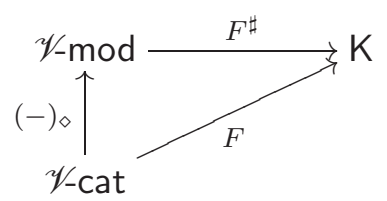

commutes. Denote by $(F f)^{*}$ the right adjoint of $F f$. Define $F^{\sharp} \mathscr{A}=F \mathscr{A}$ on objects and put $F^{\sharp}(R)$ to be the composite

$$
\left(F i_{0}\right)^{*} \cdot\left(F i_{1}\right)
$$

where $\left(i_{0}, \operatorname{Coll}(R), i_{1}\right)$ is the collage of $R$.

Then $F^{\sharp}$ preserves identities and composition by essentially the same reasoning as in the proof of Proposition 5.7 above. Using the same proof, one proves that $F^{\sharp}$ is an extension of $F$ along $(-)_{\diamond}$.

To prove that $F^{\sharp}$ is a unique extension of $F$ along $(-)_{\diamond}$, consider a 2-functor $G$ : $\mathscr{Y}$-mod $\longrightarrow \mathrm{K}$ with the property $G \cdot(-)_{\diamond}=F$. First observe that, for any $f$, from $G\left(f_{\diamond}\right)=F f$ it follows that $G\left(f^{\diamond}\right)=(F f)^{*}$, since $G$ (being a 2-functor) preserves adjunctions. 
Consider a collage $\left(i_{0}, \operatorname{Coll}(R), i_{1}\right)$ of a module $R$. Then the equalities

$$
G(R)=G\left(\left(i_{0}\right)^{\diamond} \cdot\left(i_{1}\right)_{\diamond}\right)=G\left(\left(i_{0}\right)^{\diamond}\right) \cdot G\left(\left(i_{1}\right)_{\diamond}\right)=\left(F i_{0}\right)^{*} \cdot F i_{1}=F^{\sharp}(R)
$$

prove that $G=F^{\sharp}$.

Combining the above, we arrive at our second characterisation of the existence of a relation lifting.

Corollary 5.11 (The extension theorem). For $T: \mathscr{V}$-cat $\longrightarrow \mathscr{V}$-cat, the following are equivalent:

(1) There exists a unique $\bar{T}: \mathscr{V}$-mod $\longrightarrow \mathscr{V}$-mod such that the following square

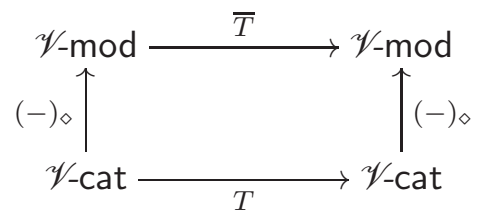

commutes.

(2) T satisfies the Beck-Chevalley Condition.

Proof. That (2) implies (1) was proved in Proposition 5.7. The uniqueness follows because the functor $(-)_{\diamond} \cdot T: \mathscr{V}$-cat $\longrightarrow \mathscr{V}$-mod has the four properties listed in Theorem 5.10. For the converse, observe that given an exact square

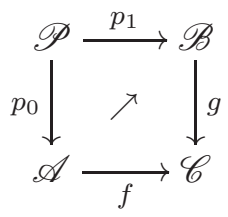

in $\mathscr{Y}$-cat, we have the equality

$$
\bar{T}\left(p_{0}\right)_{\diamond} \cdot \bar{T}\left(p_{1}\right)^{\diamond}=\bar{T}(f)^{\diamond} \cdot \bar{T}(g)_{\diamond} .
$$

Using that $\bar{T}$ preserves adjunctions we have that $\bar{T}\left(p_{1}\right)^{\diamond}=\left(T p_{1}\right)^{\diamond}$ and $\bar{T}(f)^{\diamond}=(T f)^{\diamond}$. Since $\bar{T}$ is a lifting we conclude that

$$
\left(T p_{0}\right)_{\diamond} \cdot\left(T p_{1}\right)^{\diamond}=(T f)^{\diamond} \cdot(T g)_{\diamond}
$$

in $\mathscr{Y}$-mod. Thus the functor $T$ satisfies BCC.

Remark 5.12. Explicitly, the relation lifting $\bar{T}$ of a 2 -functor $T: \mathscr{Y}$-cat $\longrightarrow \mathscr{V}$-cat is computed as follows. Given a module $R: \mathscr{A} \longrightarrow \mathscr{B}$ with the collage $\left(i_{0}, \operatorname{Coll}(R), i_{1}\right)$, we have a formula

$$
\begin{aligned}
\bar{T}(R)(B, A) & =\left(T i_{0}\right)^{\diamond} \cdot\left(T i_{1}\right)_{\diamond}(B, A) \\
& =\bigvee_{W} T \operatorname{Coll}(R)\left(W, T i_{1}(A)\right) \otimes T \operatorname{Coll}(R)\left(T i_{0}(B), W\right) \\
& =T \operatorname{Coll}(R)\left(T i_{0}(B), T i_{1}(A)\right)
\end{aligned}
$$

for every $A$ in $T \mathscr{A}$ and $B$ in $T \mathscr{B}$. 
Remark 5.13. In particular, comparing Corollary 5.11] with the extension theorem of [10, Theorem 5.3], we see that in the special case of $\mathscr{V}=2$ the lifting $\bar{T}$ of Corollary [5.11 agrees with the lifting $\bar{T}$ of [10, Theorem 5.3], due to the uniqueness of $\bar{T}$ following from the universal property of $(-)_{\diamond}: \mathscr{Y}$-cat $\longrightarrow \mathscr{K}$-mod.

Remark 5.14. The plethora of lax exact squares of Example 5.3 shows that a 2-functor satisfying BCC must preserve, for example, fully faithful and absolutely dense functors. From this, and from Corollary 5.11 above, it follows immediately that there are 2-functors $T: \mathscr{Y}$-cat $\longrightarrow \mathscr{Y}$-cat that do not admit a functorial relation lifting. See Section 6 for examples.

Remark 5.15. Combining Corollary 5.11 and Corollary 3.14 we can infer that we have at most one distributive law of a 2 -functor $T$ over $\mathbb{L}$. Indeed, if a distributive law exits, then by Corollary $3.14 T$ has a lifting $\bar{T}$ to $\mathscr{Y}$-mod. Therefore $T$ satisfies BCC. Using again Corollary 5.11 we deduce that the lifting $\bar{T}$ is unique. By the one-to-one correspondence of Corollary 3.14 we deduce that the distributive law is also unique.

The following result characterises functors admitting a relation lifting in the way akin to [15. Notice that one the phrasing suggests the following intuition when we compare relation liftings of endofunctors of sets and 2-endofunctors of $\mathscr{H}$-cat:

pullbacks $=($ the dual of $)$ cocomma squares

preservation of weak pullbacks $=($ the dual of $)$ sending cocomma squares to exact squares, preservation of epis $=$ (the dual of) preservation of fully faithful 1-cells.

Hence exact squares play precisely the rôle of weak pullbacks. Of course, every endofunctor of sets preserves epis, so preservation of epis is excluded from the conditions on the existence of the ordinary relation lifting, see [46].

The notion of cocovering cocomma squares in Condition (2) below is (the lax dual of) covering pullbacks from [15].

Proposition 5.16. For $T: \mathscr{V}$-cat $\longrightarrow \mathscr{V}$-cat, the following are equivalent:

(1) The 2-functor $T$ satisfies BCC, i.e., it preserves exact squares.

(2) The 2-functor $T$ preserves fully faithful 1-cells in $\mathscr{V}$-cat and it cocovers cocomma squares. The latter means: for every cocomma square

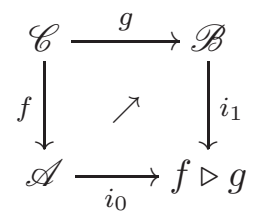


the canonical comparison can in the diagram

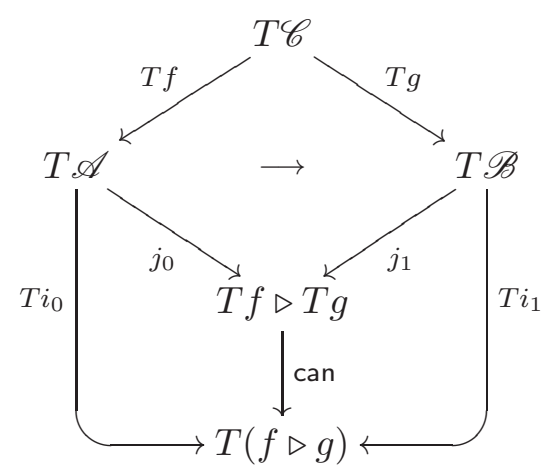

is fully faithful.

(3) The 2-functor $T$ preserves fully faithful 1-cells and it maps cocomma squares to exact squares.

Proof. (1) implies (2). By Example 5.3(3) fully faithful 1-cells can be encoded as exact squares, hence $T$ preserves them.

Consider the diagram (5.5). Then the equality

$$
(T f \triangleright T g)\left(j_{0} a, j_{1} b\right)=\bigvee_{w} T \mathscr{A}(a, T f w) \otimes T \mathscr{B}(T g w, b)
$$

holds for every $a$ in $T \mathscr{A}$ and every $b$ in $T \mathscr{B}$ since cocomma squares are exact by Example 5.3(2). But the equality

$$
\bigvee_{w} T \mathscr{A}(a, T f w) \otimes T \mathscr{B}(T g w, b)=T(f \triangleright g)\left(\operatorname{can} j_{0} a, \operatorname{can} j_{1} b\right)
$$

holds since the outer square in (5.5) is exact by assumption. Thus

$$
(T f \triangleright T g)\left(j_{0} a, j_{1} b\right)=T(f \triangleright g)\left(\operatorname{can} j_{0} a, \operatorname{can} j_{1} b\right)
$$

and we proved that can is fully faithful.

(2) implies (3). We only need to prove that the outer square in (5.5) is exact. But this follows immediately from the fact that can is fully faithful (hence $\operatorname{can}_{\diamond}$ is split mono):

$$
\left(T i_{0}\right)^{\diamond} \cdot\left(T i_{1}\right)_{\diamond}=\left(j_{0}\right)^{\diamond} \cdot \operatorname{can}^{\diamond} \cdot \operatorname{can}_{\diamond} \cdot\left(j_{1}\right)_{\diamond}=\left(j_{0}\right)^{\diamond} \cdot\left(j_{1}\right)_{\diamond}=(T f)_{\diamond} \cdot(T g)^{\diamond}
$$

(3) implies (1). Observe that the standing assumptions suffice, by Proposition 5.7 for the existence of a functorial relation lifting $\bar{T}: \mathscr{V}$-mod $\longrightarrow \mathscr{V}$-mod. Hence $T$ preserves exact squares by Corollary [5.11.

\section{EXAMPLES}

In this section, we present various examples of functors with and without BCC. Most importantly, and as a preparation for the next section, we show that the functors Set $\longrightarrow$ Set most commonly considered in coalgebra generalise to functors $\mathscr{Y}$-cat $\longrightarrow \mathscr{V}$-cat with BCC. Whereas the proof of this is more or less straightforward in most cases, it is more difficult to generalise the powerset functor. First, in the richer setting of $\mathscr{V}$-cat there are a number of choices to make in the definition, second one has to establish BCC, which does not seem to follow from general reasoning alone. Thus we give here a-to our knowledge - novel 
definition of generalised power functor on $\mathscr{K}$-cat and then establish BCC under additional assumptions on $\mathscr{V}$.

Our first example presents a functor which does not preserve fully faithful 1-cells (embeddings) and therefore, see Example 5.3(3), does not have BCC.

Example 6.1. We exhibit an example of a 2-functor that does not satisfy the BCC. To that end, we put $\mathscr{V}=2$. Recall that $\mathscr{V}$-cat is the category Pre of preorders. By Example [5.3](3), it suffices to find a locally monotone functor $T$ : Pre $\longrightarrow$ Pre that does not preserve orderembeddings (= fully faithful monotone maps). For this, let $T$ be the connected components functor, i.e., $T$ takes a preorder $\mathscr{A}$ to the discretely ordered poset of connected components of $\mathscr{A}$. The functor $T$ does not preserve the embedding $f: \mathscr{A} \longrightarrow \mathscr{B}$ indicated below.
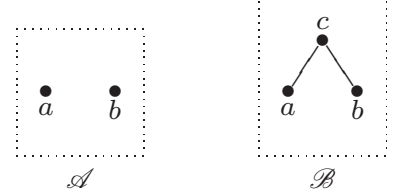

Next we present an example which preserves fully faithful 1-cells but does not have BCC. It generalises a well known example from Aczel and Mendler [2] for a functor Set $\longrightarrow$ Set which does not preserves weak pullbacks.

Example 6.2. We present an example of a 2-functor $T: \mathscr{Y}$-cat $\longrightarrow \mathscr{Y}$-cat that does preserve fully faithful 1-cells, yet it does not satisfy the BCC.

Denote, for every $\mathscr{A}$, by $\mathscr{A}^{3}$ the category $\mathscr{A} \otimes \mathscr{A} \otimes \mathscr{A}$, and denote, for every $f: \mathscr{A} \longrightarrow$ $\mathscr{B}$, by $f^{3}$ the functor $f \otimes f \otimes f$. Define $T \mathscr{A}$ to be the full subcategory of $\mathscr{A}^{3}$ spanned by objects $\left(a_{1}, a_{2}, a_{3}\right)$ such that $a_{1}=a_{2}$ or $a_{1}=a_{3}$ or $a_{2}=a_{3}$. Since, for $f: \mathscr{A} \longrightarrow \mathscr{B}$, the functor $f^{3}: \mathscr{A}^{3} \longrightarrow \mathscr{B}^{3}$ clearly restricts to a functor $T \mathscr{A} \longrightarrow T \mathscr{B}$, we have defined a 2-functor $T: \mathscr{V}$-cat $\longrightarrow \mathscr{V}$-cat.

Clearly, $T f$ is fully faithful whenever $f$ is. To prove that $T$ does not satisfy the BCC, denote by $\mathscr{I}$ the unit category $\mathscr{I}$ having one object $($ say $\star)$ and $\mathscr{I}(\star, \star)=I$. Consider $\mathscr{A}$ to be the category on two objects $a$ and $b$, with $\mathscr{A}(a, b)=\mathscr{A}(b, a)=\mathscr{A}(a, a)=\mathscr{A}(b, b)=I$. Then the commutative square

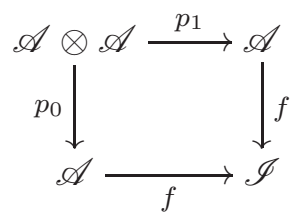

where $p_{0}$ and $p_{1}$ are projections and $f$ is the unique functor, is exact, but its image under $T$ is not.

Analogously to the "classical" case, we present a wide class of 2 -functors $T: \mathcal{Y}$-cat $\longrightarrow$ $\mathscr{Y}$-cat that satisfy the Beck-Chevalley Condition.

Example 6.3. The Kripke-polynomial 2-functors $T: \mathcal{Y}$-cat $\longrightarrow \mathscr{K}$-cat, given by the grammar

$$
T::=I d\left|\operatorname{const}_{\mathscr{X}}\right| T+T|T \otimes T| T^{\partial} \mid \mathbb{L} T
$$

are defined as follows:

(1) The 2 -functor const $\mathscr{X}$ sends every category $\mathscr{A}$ to $\mathscr{X}$ and every functor $f: \mathscr{A} \longrightarrow \mathscr{B}$ to the identity functor $I d: \mathscr{X} \longrightarrow \mathscr{X}$. 
(2) Given 2-functors $T_{1}, T_{2}: \mathscr{Y}$-cat $\longrightarrow \mathscr{Y}$-cat, the 2-functors $T_{1}+T_{2}$ and $T_{1} \otimes T_{2}$, send a category $\mathscr{A}$ to $T_{1} \mathscr{A}+T_{2} \mathscr{A}$ and $T_{1} \mathscr{A} \otimes T_{2} \mathscr{A}$, respectively. For a functor $f: \mathscr{A} \longrightarrow \mathscr{B}$, the values are $T_{1} f+T_{2} f$ and $T_{1} f \otimes T_{2} f$, respectively.

(3) The 2 -functor $T^{\partial}$ (the dual of the 2 -functor $T$ ) is defined as the following composite

$$
\begin{aligned}
& \mathscr{Y} \text {-cat } \stackrel{(-)^{o p}}{\longrightarrow} \mathscr{Y} \text {-cat }{ }^{c o} \stackrel{T^{c o}}{\longrightarrow} \mathscr{Y} \text {-cat }{ }^{c o} \stackrel{(-)^{o p}}{\longrightarrow} \mathscr{Y} \text {-cat } \\
& \mathscr{A} \longmapsto \mathscr{A}^{o p} \longmapsto T\left(\mathscr{A}^{o p}\right) \longmapsto\left(T\left(\mathscr{A}^{o p}\right)\right)^{o p}
\end{aligned}
$$

where $\mathscr{Y}$-cat ${ }^{c o}$ is the Pre-category $\mathscr{Y}$-cat with just the 2 -cells reversed, i.e., the equality

$$
\mathscr{Y}_{\text {-cat }}{ }^{c o}(\mathscr{A}, \mathscr{B})=(\mathscr{Y} \text {-cat }(\mathscr{A}, \mathscr{B}))^{o p}
$$

holds for all categories $\mathscr{A}$ and $\mathscr{B}$. The functor $T^{c o}: \mathscr{V}$-cat ${ }^{c o} \longrightarrow \mathscr{Y}$-cat ${ }^{c o}$ acts exactly as $T$ on 0 -cells and 1 -cells. Using $\mathscr{Y}$-cat ${ }^{c o}$ is a technicality needed to make $(-)^{o p}$ a 2 -functor. Hence it is easy to check that $T^{\partial}$ is a 2 -functor.

(4) The 2 -functor $\mathbb{L}$ sends $\mathscr{A}$ to $\left[\mathscr{A}^{o p}, \mathscr{V}\right]$ and $f: \mathscr{A} \longrightarrow \mathscr{B}$ is sent to the left Kan extension along $f^{o p}$. In particular, we have an adjunction

$$
\mathbb{L} f \dashv\left[f^{o p}, \mathscr{V}\right]:\left[\mathscr{B}^{o p}, \mathscr{V}\right] \longrightarrow\left[\mathscr{A}^{o p}, \mathscr{V}\right]
$$

In a formula, we have

$$
\llbracket f: A \mapsto\left(b \mapsto \bigvee_{a} A a \otimes \mathscr{B}(b, f a)\right)
$$

It should be noted that $\mathbb{L}$ is a genuine 2-functor, it preserves identities and composition on the nose since we enrich in a quantale.

Proposition 6.4. The Kripke-polynomial functors from Example 6.3 have BCC.

Proof. Consider an exact lax square

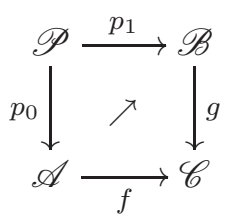

in $\mathscr{Y}$-cat. We prove by structural induction that every Kripke-polynomial $T: \mathscr{V}$-cat $\longrightarrow \mathscr{Y}$-cat preserves exactness of the above square.

(1) The 2-functor $T=I d$ satisfies preserves the exactness of (6.1) trivially.

(2) The 2-functor const $\mathscr{X}$ preserves the exactness of (6.1), since the image of square (6.1) under const $\mathscr{X}$ is the square

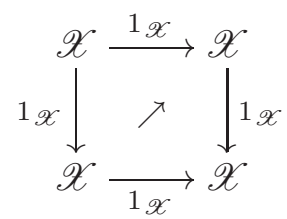

where the comparison is the identity. This is an exact square (it is a Yoneda square).

(3) Suppose both $T_{1}$ and $T_{2}$ preserve the exactness of (6.1). We prove that $T_{1}+T_{2}$ preserves the exactness of (6.1). 
The image of (6.1) under $T_{1}+T_{2}$ is

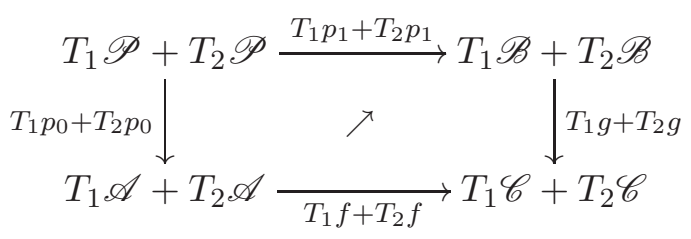

The assertion follows from the fact that coproducts are disjoint in $\mathscr{Y}$-cat.

(4) Suppose both $T_{1}$ and $T_{2}$ preserve the exactness of the square (6.1). We prove that $T_{1} \otimes T_{2}$ preserves the exactness of the square (6.1).

The image of (6.1) under $T_{1} \otimes T_{2}$ is

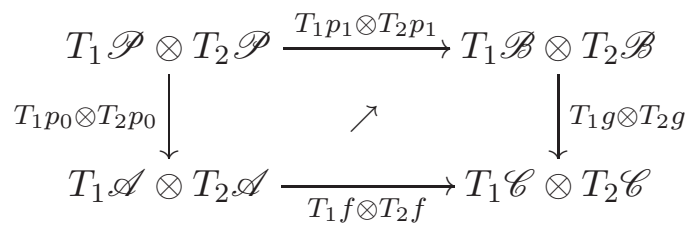

and the latter square is exact due to the following equations

$$
\begin{aligned}
&\left(T_{1} \mathscr{C} \otimes T_{2} \mathscr{C}\right)\left(\left(T_{1} f \otimes T_{2} f\right)\left(a, a^{\prime}\right),\left(T_{1} g \otimes T_{2} g\right)\left(b, b^{\prime}\right)\right) \\
&= T_{1} \mathscr{C}\left(T_{1} f a, T_{1} g b\right) \otimes T_{2} \mathscr{C}\left(T_{2} f a^{\prime}, T_{2} g b^{\prime}\right) \\
&=\left(\bigvee_{w} T_{1} \mathscr{A}\left(a, T_{1} p_{0} w\right) \otimes T_{1} \mathscr{B}\left(T_{1} p_{1} w, b\right)\right) \otimes\left(\bigvee_{w^{\prime}} T_{2} \mathscr{A}\left(a^{\prime}, T_{2} p_{0} w^{\prime}\right) \otimes T_{2} \mathscr{B}\left(T_{2} p_{1} w^{\prime}, b^{\prime}\right)\right) \\
&= \bigvee_{w, w^{\prime}}\left(T_{1} \mathscr{A}\left(a, T_{1} p_{0} w\right) \otimes T_{1} \mathscr{B}\left(T_{1} p_{1} w, b\right) \otimes T_{2} \mathscr{A}\left(a^{\prime}, T_{2} p_{0} w^{\prime}\right) \otimes T_{2} \mathscr{B}\left(T_{2} p_{1} w^{\prime}, b^{\prime}\right)\right) \\
&= \bigvee_{\left(w, w^{\prime}\right)}\left(\left(T_{1} \mathscr{A} \otimes T_{2} \mathscr{A}\right)\left(\left(a, a^{\prime}\right),\left(T_{1} p_{0} \otimes T_{2} p_{0}\right)\left(w, w^{\prime}\right)\right) \otimes\left(T_{1} \mathscr{B} \otimes T_{2} \mathscr{B}\right)\left(\left(T_{1} p_{1} \otimes T_{2} p_{1}\right)\left(w, w^{\prime}\right),\left(b, b^{\prime}\right)\right)\right)
\end{aligned}
$$

where we have used induction assumptions and the properties of $\otimes$ in $\mathscr{V}$.

(5) Suppose $T$ preserves the exactness of the square (6.1). We prove that its dual $T^{\partial}$ preserves the exactness of the square (6.1).

The square

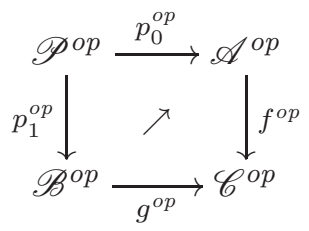

is exact by Example 5.4, since (6.1) is exact. By assumption, the square

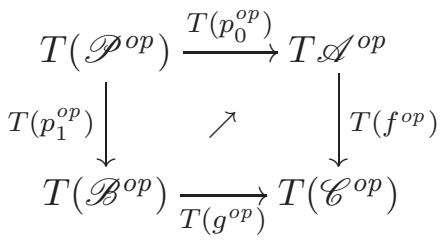


is exact. Finally, the square

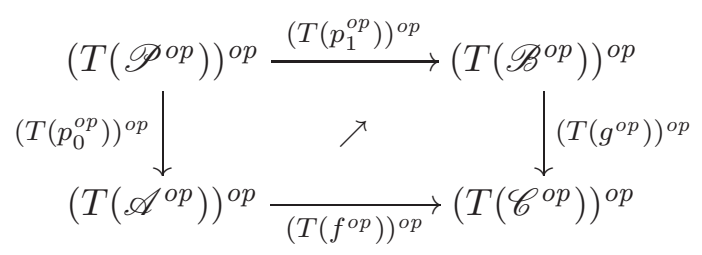

is exact by Example 5.4 and this is what we were supposed to prove.

(6) Suppose that $T$ preserves the exactness of the square (6.1). We prove that $\mathbb{L} T$ does preserve it again.

It suffices to prove that $\mathbb{L}$ satisfies the Beck-Chevalley Condition. The image of square (6.1) under $\mathbb{L}$ is the square

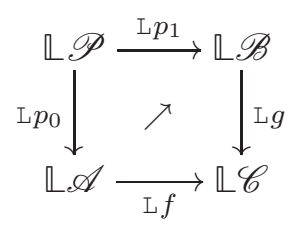

We employ Example 5.5; both $\mathbb{L} f$ and $\mathbb{L} p_{1}$ are left adjoints with $(\mathbb{L} f)^{r}=\left[f^{o p}, \mathscr{V}\right]$ and $\left(\mathbb{L} p_{1}\right)^{r}=\left[p_{1}^{o p}, \mathscr{V}\right]$. Hence it suffices to prove that there is an isomorphism

$$
\llbracket p_{0} \cdot\left[p_{1}^{o p}, \mathscr{V}\right] \cong\left[f^{o p}, \mathscr{V}\right] \cdot \llbracket g
$$

Moreover, by the density of representables, i.e., of functors of the form $\mathscr{B}\left(-, b_{0}\right)$ in $\mathbb{L} \mathscr{B}$, and by the fact that all the functors $\mathbb{L} p_{0},\left[p_{1}^{o p}, 2\right],\left[f^{o p}, 2\right], \mathbb{L} g$ preserve colimits (since they all are left adjoints), it suffices to prove that

$$
\left(\mathbb{L} p_{0} \cdot\left[p_{1}^{o p}, \mathscr{V}\right]\right)\left(\mathscr{B}\left(-, b_{0}\right)\right) \cong\left(\left[f^{o p}, \mathscr{V}\right] \cdot \llbracket g\right)\left(\mathscr{B}\left(-, b_{0}\right)\right)
$$

holds for all $b_{0}$.

The left-hand side is isomorphic to

$$
\llbracket p_{0}\left(\mathscr{B}\left(p_{1}-, b_{0}\right)\right)=a \mapsto \bigvee_{w} \mathscr{A}\left(a, p_{0} w\right) \otimes \mathscr{B}\left(p_{1} w, b_{0}\right)
$$

By exactness of (6.1), this means that

$$
\llbracket p_{0}\left(\mathscr{B}\left(p_{1}-, b_{0}\right)\right)=a \mapsto \mathscr{C}\left(f a, g b_{0}\right)
$$

Observe further that

$$
(\mathbb{L} g)\left(\mathscr{B}\left(-, b_{0}\right)\right)=c \mapsto \bigvee_{b} \mathscr{C}(c, g b) \otimes \mathscr{B}\left(b, b_{0}\right)
$$

hence

$$
(\mathbb{L} g)\left(\mathscr{B}\left(-, b_{0}\right)\right)=c \mapsto \mathscr{C}\left(c, g b_{0}\right)
$$

by the Yoneda Lemma.

The right hand side of (6.2) is therefore isomorphic to

$$
\left(\left[f^{o p}, \mathscr{V}\right] \cdot \mathbb{L} g\right)\left(\mathscr{B}\left(-, b_{0}\right)\right)=\left[f^{o p}, \mathscr{V}\right]\left(c \mapsto \mathscr{C}\left(c, g b_{0}\right)\right)=a \mapsto \mathscr{C}\left(f a, g b_{0}\right)
$$

Hence the isomorphism in (6.2) takes place, and therefore $\mathbb{L}$ preserves exactness of the square (6.1). 
We write $\mathbb{U}$ for $\mathbb{L}^{\partial}$. In the case $\mathscr{V}=2$, the two functors $\mathbb{L}$ and $\mathbb{U}$ are the usual lowerand upper-sets functors. In domain theory this distinction is known under the names of Hoare and Smyth powerdomains [1, Prop 6.2.12].

Next we show how to use the formula for the relation lifting from Remark 5.12 in order to compute the relation lifting of $\mathbb{L}$ and $\mathbb{U}$.

Example 6.5 (Relation liftings of $\mathbb{L}$ and $\mathbb{U}$ ). By Example 6.3 , the 2 -functor $\mathbb{L}: \mathscr{Y}$-cat $\longrightarrow$ $\mathscr{V}$-cat satisfies the BCC. Since $\mathbb{L} f \dashv\left[f^{o p}, \mathscr{V}\right]$, the relation lifting $\mathbb{\mathbb { L }}$ of $\mathbb{L}$ is given by the formula

$$
\begin{aligned}
\overline{\mathbb{L}}(R)(B, A) & =\mathbb{L} \operatorname{Coll}(R)\left(\mathbb{L} i_{0}(B), \mathbb{L} i_{1}(A)\right) \\
& =\mathbb{L} \mathscr{B}\left(B,\left[i_{0}^{o p}, \mathscr{V}\right] \cdot \mathbb{L} i_{1}(A)\right) \\
& =\bigwedge_{b}\left[B b, \bigvee_{a} \operatorname{Coll}(R)\left(i_{0}(b), i_{1}(a)\right) \otimes A a\right] \\
& =\bigwedge_{b}\left[B b, \bigvee_{a} R(b, a) \otimes A a\right]
\end{aligned}
$$

for every $R: \mathscr{A} \longrightarrow \mathscr{B}, A: \mathscr{A}^{o p} \longrightarrow \mathscr{V}$ and $B: \mathscr{B}^{o p} \longrightarrow \mathscr{V}$.

The dual $\mathbb{L}^{\partial}=\mathbb{U}$ is explicitly given by $\mathbb{U} \mathscr{A}=[\mathscr{A}, \mathscr{V}]^{o p}$ and $\mathbb{U} f: \mathbb{U} \mathscr{A} \longrightarrow \mathbb{U} \mathscr{B}$ is defined, for $f: \mathscr{A} \longrightarrow \mathscr{B}$, as the right Kan extension along $f$. Hence we have an adjunction $[f, \mathscr{V}]^{o p} \dashv \mathbb{U} f$. Therefore the relation lifting $\overline{\mathbb{U}}$ of $\mathbb{U}$ is given by the formula

$$
\begin{aligned}
\overline{\mathbb{U}}(R)(B, A) & =\mathbb{U} \operatorname{Coll}(R)\left(\mathbb{U} i_{0}(B), \mathbb{U} i_{1}(A)\right) \\
& =\mathbb{U} \mathscr{A}\left(\left[i_{1}, \mathscr{V}\right]^{o p} \mathbb{U} i_{0}(B), A\right) \\
& =[\mathscr{A}, \mathscr{V}]\left(A,\left[i_{1}, \mathscr{V}\right]^{o p} \mathbb{U} i_{0}(B)\right) \\
& =\bigwedge_{a}\left[A a, \bigvee_{b} \operatorname{Coll}(R)\left(i_{0}(b), i_{1}(a)\right) \otimes B b\right] \\
& =\bigwedge_{a}\left[A a, \bigvee_{b} R(b, a) \otimes B b\right]
\end{aligned}
$$

for every $R: \mathscr{A} \longrightarrow \mathscr{B}, A: \mathscr{A} \longrightarrow \mathscr{V}$ and $B: \mathscr{B} \longrightarrow \mathscr{V}$.

Remark 6.6. For further reference, given $R: \mathscr{A} \longrightarrow \mathscr{B}$, we have

$$
\begin{aligned}
& \overline{\mathbb{Z}}(R)(B, A)=\bigwedge_{b}\left[B b, \bigvee_{a} R(b, a) \otimes A a\right] \\
& \overline{\mathbb{U}}(R)(B, A)=\bigwedge_{a}\left[A a, \bigvee_{b} R(b, a) \otimes B b\right]
\end{aligned}
$$

Note that in the case $\mathscr{V}=2$ these two formulas become, as expected,

$$
\begin{aligned}
& \overline{\mathbb{L}}(R)(B, A)=\forall b \in B . \exists a \in A . R(b, a) \\
& \overline{\mathbb{U}}(R)(B, A)=\forall a \in A . \exists b \in B . R(b, a)
\end{aligned}
$$

Finally, we come to generalising the powerset functor. We start from the point of view that in the case $\mathscr{V}=2$, the natural generalisation of the powerset functor to a functor $\mathbb{P}: \mathscr{Y}$-cat $\longrightarrow \mathscr{V}$-cat is given as follows. On objects, a preorder $\mathscr{A}$ is mapped to the set of all subsets of the carrier of $\mathscr{A}$, ordered by the so-called Egli-Milner order

$$
B \leq_{E M} A \Leftrightarrow\left\{\begin{array}{l}
\forall b \in B . \exists a \in A . b \leq_{\mathscr{A}} a \\
\forall a \in A . \exists b \in B . b \leq_{\mathscr{A}} a
\end{array}\right.
$$


The similarity with (6.4) is not a coincidence, but we emphasise that for $\mathbb{P}$ we consider all subsets, not only upper or lower subsets. On arrows $\mathbb{P}$ is given by direct image.

It is clear that $\mathbb{P}$ is a straightforward generalisation of the powerset functor. Conceptually, only the choice of the Egli-Milner order needs justification. One such justification has been given in [47] where it was shown that the definition of $\mathbb{P}$ is just a special instance of a general construction of lifting functors from Set to Pre (that paper discusses the category Pos of posets instead of the category Pre of preorders, but the construction and argument remain the same): In a nutshell, in the same way as the powerset functor is a quotient of the list functor on Set by a set $E$ of equations, the Egli-Milner order arises from quotienting (in Pre) the list functor on Pre by the same set $E$ of equations.

Another justification, which will play a role in the next section, comes from the observation that the Pos-collapse of $\mathbb{P}$ is the convex powerspace functor, which follows from 1 , Prop 6.2.5.6]. The convex power functor is known in domain theory as the Plotkin powerdomain, see eg [1], and has been studied in a coalgebraic context in [38. In this line of research it is well-known that the convex powerset provides the Kripke semantics for negation-free modal logic in the same way as the usual powerset provides the Kripke semantics for classical modal logic.

Coming back to the task of defining $\mathbb{P}: \mathscr{Y}$-cat $\longrightarrow \mathscr{Y}$-cat, we want to generalise (6.5) and notice the similarity with the right hand sides of (6.4), which we know to be generalised by the right hand sides of (6.3). We are thus led to (6.6) below.

Example 6.7. The generalised power 2-functor $\mathbb{P}: \mathscr{V}$-cat $\longrightarrow \mathscr{V}$-cat is defined as follows. We start with some terminology and notation. Given a $\mathscr{V}$-category $\mathscr{A}$, a $\mathscr{V}$-subset of $\mathscr{A}$ is an arbitrary $\mathscr{V}$-functor from the discrete underlying $\mathscr{V}$-category $|\mathscr{A}|$ to $\mathscr{V}$. Recall that $|\mathscr{A}|$ has the same objects as $\mathscr{A}$ and

$$
|\mathscr{A}|\left(a, a^{\prime}\right)= \begin{cases}I, & \text { if } a=a^{\prime} \\ \perp, & \text { else. }\end{cases}
$$

Hence a $\mathscr{V}$-subset $\varphi:|\mathscr{A}| \longrightarrow \mathscr{V}$ of $\mathscr{A}$ is a mere collection $\{\varphi(a)\}$ of elements of $\mathscr{V}_{o}$, indexed by objects of $\mathscr{A}$. For a $\mathscr{V}$-subset $\varphi$ of $\mathscr{A}$, we define

$$
\varphi^{\uparrow}:|\mathscr{A}| \longrightarrow \mathscr{V} \quad \text { and } \quad \varphi^{\downarrow}:|\mathscr{A}| \longrightarrow \mathscr{V}
$$

by the formulas

$$
\varphi^{\uparrow}(a)=\bigvee_{a^{\prime}} \varphi\left(a^{\prime}\right) \otimes \mathscr{A}\left(a^{\prime}, a\right), \quad \varphi^{\downarrow}(a)=\bigvee_{a^{\prime}} \varphi\left(a^{\prime}\right) \otimes \mathscr{A}\left(a, a^{\prime}\right)
$$

Intuitively, $\varphi^{\uparrow}$ is the "upper-set" generated by $\varphi$, considered as a mere $\mathscr{V}$-subset of $\mathscr{A}$. Similarly, $\varphi^{\downarrow}$ is the "lower-set" generated by $\varphi$.

The 2-functor $\mathbb{P}: \mathscr{V}$-cat $\longrightarrow \mathscr{V}$-cat is now defined as follows:

(1) The objects of $\mathbb{P} \mathscr{A}$ are arbitrary $\mathscr{V}$-subsets $\varphi:|\mathscr{A}| \longrightarrow \mathscr{V}$ of $\mathscr{A}$. For any $\varphi, \psi:|\mathscr{A}| \longrightarrow$ $\mathscr{V}$ put

$$
\mathbb{P} \mathscr{A}(\varphi, \psi)=[|\mathscr{A}|, \mathscr{V}]\left(\varphi, \psi^{\downarrow}\right) \otimes[|\mathscr{A}|, \mathscr{V}]\left(\psi, \varphi^{\uparrow}\right)
$$

or, in a detailed formula, by

$$
\mathbb{P} \mathscr{A}(\varphi, \psi)=\bigwedge_{a}\left[\varphi(a), \bigvee_{a^{\prime}} \psi\left(a^{\prime}\right) \otimes \mathscr{A}\left(a, a^{\prime}\right)\right] \otimes \bigwedge_{a^{\prime}}\left[\psi\left(a^{\prime}\right), \bigvee_{a} \varphi(a) \otimes \mathscr{A}\left(a, a^{\prime}\right)\right]
$$

that can be perceived as the "Egli-Milner condition in the $\mathscr{V}$-setting". 
(2) Given a $\mathscr{V}$-functor $f: \mathscr{A} \longrightarrow \mathscr{B}$ and $\mathscr{V}$-subset $\varphi:|\mathscr{A}| \longrightarrow \mathscr{V}$, define $\operatorname{Pf}(\varphi):|\mathscr{B}| \longrightarrow \mathscr{V}$ by

$$
b \mapsto \bigvee_{a}|\mathscr{B}|(f a, b) \otimes \varphi a .
$$

In other words, $\mathbb{P} f(\varphi)$ is the value of a left Kan extension of $\varphi$ along $|f|:|\mathscr{A}| \longrightarrow|\mathscr{B}|$. In particular, the equality

$$
[|B|, \mathscr{V}](\mathbb{P} f(\varphi), \psi)=[|\mathscr{A}|, \mathscr{V}](\varphi, \psi \cdot|f|)
$$

holds for all $\varphi:|\mathscr{A}| \longrightarrow \mathscr{V}, \psi:|\mathscr{B}| \longrightarrow \mathscr{V}$.

Long but standard lattice-theoretical computations show that $\mathbb{P}$ is indeed a 2 -functor. Notice that when $\mathscr{V}$ is 2 , we obtain the power 2-functor on Pre defined in [10, Example 6.3].

Moreover, if $\mathscr{V}$ is such that $\otimes$ is $\wedge$, then $\mathbb{P}$ satisfies the Beck-Chevalley Condition. Consider an exact square

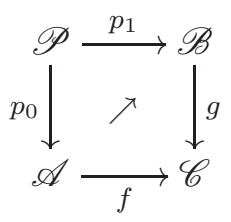

We have to show that the equality

$$
\mathbb{P} \mathscr{C}(\mathbb{P} f(\varphi), \mathbb{P} g(\psi))=\bigvee_{\theta} \mathbb{P} \mathscr{A}\left(\varphi, \mathbb{P} p_{0}(\theta)\right) \otimes \mathbb{P} \mathscr{B}\left(\mathbb{P} p_{1}(\theta), \psi\right)
$$

holds for all $\varphi$ and $\psi$. By (5.2), the inequality

$$
\mathbb{P} \mathscr{C}(\mathbb{P} f(\varphi), \mathbb{P} g(\psi)) \geq \bigvee_{\theta} \mathbb{P} \mathscr{A}\left(\varphi, \mathbb{P} p_{0}(\theta)\right) \otimes \mathbb{P} \mathscr{B}\left(\mathbb{P} p_{1}(\theta), \psi\right)
$$

holds always, hence we only need to prove the reversed inequality. To that end, consider the $\mathscr{V}$-subset $\theta:|\mathscr{P}| \longrightarrow \mathscr{V}$, defined by

$$
\theta(w)=\varphi^{\uparrow}\left(p_{0} w\right) \wedge \psi^{\downarrow}\left(p_{1} w\right)
$$

Since the equalities

$$
\begin{aligned}
\mathbb{P} \mathscr{C}(\mathbb{P} f(\varphi), \mathbb{P} g(\psi)) & =[|\mathscr{C}|, \mathscr{V}]\left(\mathbb{P} f(\varphi), \mathbb{P} g(\psi)^{\downarrow}\right) \otimes[|\mathscr{C}|, \mathscr{V}]\left(\mathbb{P} g(\psi), \mathbb{P} f(\varphi)^{\uparrow}\right) \\
& =[|\mathscr{A}|, \mathscr{V}]\left(\varphi, \mathbb{P} g(\psi)^{\downarrow} \cdot|f|\right) \otimes[|\mathscr{B}|, \mathscr{V}]\left(\psi, \mathbb{P} f(\varphi)^{\uparrow} \cdot|f|\right) \\
& =\bigwedge_{a}\left[\varphi(a), \bigvee_{b} \mathscr{C}(f a, g b) \otimes \psi(b)\right] \otimes \bigwedge_{b}\left[\psi(b), \bigvee_{a} \mathscr{C}(f a, g b) \otimes \varphi(a)\right]
\end{aligned}
$$

and

$$
\bigvee_{\theta} \mathbb{P} \mathscr{A}\left(\varphi, \mathbb{P} p_{0}(\theta)\right) \otimes \mathbb{P} \mathscr{B}\left(\mathbb{P} p_{1}(\theta), \psi\right)=\bigvee_{\theta} \mathbb{P} \mathscr{A}\left(\varphi, \mathbb{P} p_{0}(\theta)\right) \otimes \mathbb{P} \mathscr{B}\left(\theta, \psi \cdot\left|p_{1}\right|\right)
$$

hold by definition, it suffices to prove the inequalities

$$
\bigwedge_{a}\left[\varphi(a), \bigvee_{b} \psi(b) \otimes \mathscr{C}(f a, g b)\right] \leq \bigwedge_{a}\left[\varphi(a), \bigvee_{w} \theta(w) \otimes \mathscr{A}\left(a, p_{0} w\right)\right] \leq \mathbb{P} \mathscr{A}\left(\varphi, \mathbb{P} p_{0}(\theta)\right)
$$

and

$$
\bigwedge_{b}\left[\psi(b), \bigvee_{a} \mathscr{C}(f a, g b) \otimes \varphi(a)\right] \leq \bigwedge_{b}\left[\psi(b), \bigvee_{w} \theta(w) \otimes \mathscr{B}\left(p_{1} w, b\right)\right] \leq \mathbb{P} \mathscr{B}\left(\theta, \psi \cdot\left|p_{1}\right|\right)
$$


The first inequality in (6.8) is proved using the equality $[x, x \otimes y]=[x, y]$ in $\mathscr{V}_{o}$ (that holds since $\otimes$ is assumed to be $\wedge)$ and the exactness equality $\mathscr{C}(f a, g b)=\bigvee_{w} \mathscr{A}\left(a, p_{0} w\right) \otimes$ $\mathscr{B}\left(p_{1} w, b\right)$, the second inequality in (6.8) follows from $\otimes$ being $\wedge$. The inequalities (6.9) follow analogously.

This finishes the proof that, for $\mathscr{V}$ with $\otimes=\wedge$, it satisfies the Beck-Chevalley Condition. We recall that $\otimes=\wedge$ holds in generalised ultrametric spaces but not in generalised metric spaces.

We conclude by showing that the relation lifting of $\mathbb{P}$ indeed combines the two formulas for $\mathbb{L}$ and $\mathbb{U}$ from $(6.3)$.

Example 6.8 (Relation lifting of $\mathbb{P}$ ). Let $\mathscr{V}$ be a quantale such that $\otimes=\wedge$. By Example 6.7 we have that $\mathbb{P}$ satisfies the BCC. For all $A:|\mathscr{A}| \longrightarrow \mathscr{V}$ and $B:|\mathscr{B}| \longrightarrow \mathscr{V}$, the relation lifting $\overline{\mathbb{P}}$ of $\mathbb{P}$ is given by

$$
\begin{aligned}
\overline{\mathbb{P}}(R)(B, A)= & \mathbb{P C o l l}(R)\left(\mathbb{P} i_{0}(B), \mathbb{P} i_{1}(A)\right. \\
= & \bigwedge_{y \in \operatorname{Coll}(R)}\left[\mathbb{P} i_{0}(B)(y), \bigvee_{x \in \operatorname{Coll}(R)} \mathbb{P} i_{1}(A)(x) \otimes \operatorname{Coll}(R)(y, x)\right] \otimes \\
= & \bigwedge_{x \in \operatorname{Coll}(R)}\left[\mathbb{P} i_{1}(A)(x), \bigvee_{y \in \operatorname{Coll}(R)} \mathbb{P} i_{0}(B)(y) \otimes \operatorname{Coll}(R)(y, x)\right] \\
& \bigwedge_{y \in \mathscr{A}}\left[B(y), \bigvee_{y \in \operatorname{Coll}(R)} \mathbb{P} i_{1}(A)(x) \otimes \operatorname{Coll}(R)(y, x)\right] \wedge \\
& \bigwedge_{x \in \mathscr{A}}\left[\mathbb{P} i_{1}(A)(x), \bigvee_{y \in \operatorname{Coll}(R)} \mathbb{P} i_{1}(A)(x) \otimes \operatorname{Coll}(R)(y, x)\right] \wedge \\
& \bigwedge_{x \in \mathscr{B}}\left[\perp, \bigvee_{y \in \operatorname{Coll}(R)} \mathbb{P} i_{0}(B)(y) \otimes \operatorname{Coll}(R)(y, x)\right] \\
= & \bigwedge_{b \in \mathscr{B}}\left[B(b), \bigvee_{a \in \mathscr{A}} A(a) \otimes R(b, a)\right] \wedge \bigwedge_{a \in \mathscr{A}}\left[A(a), \bigvee_{b \in \mathscr{B}} B(b) \otimes R(b, a)\right]
\end{aligned}
$$

\section{Moss's COVER MODALITy $\nabla$ OVER $\mathscr{Y}$-cat}

In this section we apply the material collected so far and investigate the semantics of Moss's cover modality $\nabla$. We first extend the standard notion of bisimilarity to coalgebras for functors $T$ on $\mathscr{Y}$-cat and then show that $\nabla$ is invariant under bisimilarity if $T$ satisfies the Beck-Chevalley Condition.

\section{A. Coalgebras and bisimilarity.}

Definition 7.1. A $T$-coalgebra is a $\mathscr{V}$-functor $\xi: \mathscr{X} \longrightarrow T \mathscr{X}$. Elements of $\mathscr{X}$ are called states and $\xi$ is the transition structure. A coalgebra morphism from $\xi: \mathscr{X} \longrightarrow T \mathscr{X}$ to $\xi^{\prime}: \mathscr{X}^{\prime} \longrightarrow T \mathscr{X}^{\prime}$ is $\mathscr{V}$-functor $f: \mathscr{X} \longrightarrow \mathscr{X}^{\prime}$ such that $\xi^{\prime} \cdot f=T f \cdot \xi$. The category of $T$ coalgebras is denoted by $\operatorname{Coalg}(T)$ and we write $U: \operatorname{Coalg}(T) \longrightarrow \mathscr{V}$-cat and $V: \mathscr{Y}$-cat $\longrightarrow$ Set for the respective forgetful functors. 
The current setting is rich in examples. For functors $T$ we can choose at least those of Example 6.3. For $\mathscr{V}$ we can choose any commutative quantale. So we certainly encompass coalgebras over posets and over ultrametric spaces, which play a fundamental role in domain theory and the semantics of programming languages [1, 6]. In a specific coalgebraic context, they were investigated eg in [40, 50, 24, 27, 32, 4].

The following example illustrates in the case of generalised metric spaces the effect of the coalgebra structure being non-expansive.

Example 7.2. Consider $\mathscr{X} \in \mathrm{GMet}$ and $\xi: \mathscr{X} \longrightarrow \mathbb{L} \mathscr{X}$. The requirement that $\xi$ is a $\mathscr{V}$-functor, ie a non-expansive map, gives us

$$
\begin{aligned}
X\left(x, x^{\prime}\right) & \geq_{\mathrm{R}} \mathbb{L} \mathscr{X}\left(\xi(x), \xi\left(x^{\prime}\right)\right) \\
& =\left[\mathscr{X}^{o p}, \mathscr{V}\right]\left(\xi(x)(y), \xi\left(x^{\prime}\right)(y)\right) \\
& =\bigwedge_{y \in \mathscr{X}}\left[\xi(x)(y), \xi\left(x^{\prime}\right)(y)\right] \\
& =\sup _{y \in \mathscr{X}}\left(\xi\left(x^{\prime}\right)(y)-\xi(x)(y)\right)
\end{aligned}
$$

This is equivalent to

$$
\forall y \in \mathscr{X} \cdot \xi\left(x^{\prime}\right)(y) \leq_{\mathrm{R}} X\left(x, x^{\prime}\right)+\xi(x)(y) .
$$

Similarly, for $\xi: \mathscr{X} \longrightarrow \mathbb{U} \mathscr{X}$, we obtain

$$
\forall y \in \mathscr{X} \cdot \xi(x)(y) \leq_{\mathrm{R}} X\left(x, x^{\prime}\right)+\xi\left(x^{\prime}\right)(y) .
$$

We see that non-expansiveness of $\xi$ corresponds to a triangle-inequality relating "internal moves in $\mathscr{X}$ " with "external moves in the coalgebra". Note that the direction of the "internal moves" (dotted below) is different in both cases, in a picture:

$$
\xi: \mathscr{X} \longrightarrow \mathbb{L} \mathscr{X}
$$

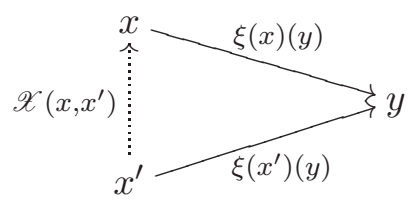

$\xi: \mathscr{X} \longrightarrow \mathbb{U} \mathscr{X}$

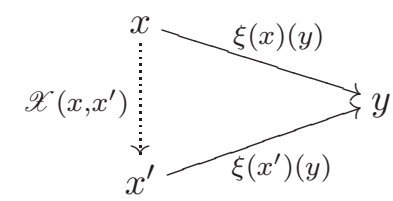

Any object $\mathscr{A}$ of $\mathscr{V}$-cat induces a coalgebra $\mathscr{A} \longrightarrow \mathbb{L} \mathscr{A}$ via the correspondence of $\mathscr{A}(-,-)$ : $\mathscr{A}^{o p} \otimes \mathscr{A} \longrightarrow \mathscr{V}$ with $\mathscr{A} \longrightarrow\left[\mathscr{A}^{o p}, \mathscr{V}\right]=\mathbb{L}(\mathscr{A})$ and a coalgebra $\mathscr{A} \longrightarrow \mathbb{U}$ via the correspondence of $\mathscr{A}(-,-): \mathscr{A}^{o p} \otimes \mathscr{A} \longrightarrow \mathscr{V}$ with $\mathscr{A} \longrightarrow[\mathscr{A}, \mathscr{V}]^{o p}=\left(\mathbb{L}\left(\mathscr{A}^{o p}\right)\right)^{o p}=\mathbb{U}(\mathscr{A})$.

Example 7.3. In case of $\mathscr{A}$ being $A^{\infty}$ as in item 3 of Example 2.19 we have the following instances of the previous example.

(1) The coalgebra $\mathscr{A} \longrightarrow \mathbb{L} \mathscr{A}$ maps a state $w$ to the predicate $A^{\infty}(-, w)$. Intuitively, from $w$ one can move at no costs to any prefix $v \leq w$ and from there to any extension $v^{\prime}$ of $v$ at cost $\sum_{i=|v|+1}^{\left|v^{\prime}\right|}$. This also illustrates how $\mathbb{L}$ generalises the lower-sets functor known from preorders, ie $\mathscr{V}=2$.

(2) The coalgebra $\mathscr{A} \longrightarrow \mathbb{U} \mathscr{A}$ maps a state $w$ to the predicate $A^{\infty}(w,-)$. Intuitively, from $w$ one can move at no costs to any extension $v$ with $w \leq v$, but one can move to other $v$ only at a cost which measures "the amount of information lost by erasing elements from $w$ ". Note how $\mathbb{U}$ generalises the upper-sets functor known from preorders (or posets). 
In the case of coalgebras over Set there are different ways to define bisimilarity for coalgebras. One can define it via the notion of a largest bisimulation, which does exists if $T$ preserves weak pullbacks (=satisfies BCC), see [41] for an introduction and [42] for a recent survey. A more conceptual approach defines bisimilarity as what remains invariant under coalgebra morphisms. This idea can be made precise in different ways, eg via the final coalgebra, via cospans, or as in the next definition taken from [30]. An additional benefit of this definition is that it does not depend on special properties of $T$ or $\mathscr{V}$-cat. Recall the forgetful functors $U: \operatorname{Coalg}(T) \longrightarrow \mathscr{V}$-cat and $V: \mathscr{V}$-cat $\longrightarrow$ Set introduced in Definition 7.1 .

Definition 7.4. Bisimilarity, or behavioural equivalence, is the smallest equivalence relation on elements of coalgebras generated by pairs

$$
(x, V U f(x))
$$

where $x$ is an element of a coalgebra and $f$ is a coalgebra morphism.

The carrier of that relation is a proper class as we allow us to compare states from any two given coalgebras. Next, we show that bisimilarity is the equivalence relation classified by the final coalgebra.

Proposition 7.5. If the final T-coalgebra exists, then its carrier is given by colim $V U$.

Proof. If the final $T$-coalgebra exists, then it is colim $I d$ where $I d$ is the identity functor on Coalg $(T)$, see [33, Chapter X.1]. Since $U$ preserves colimits, we have $U(\operatorname{colim} I d)=\operatorname{colim} U$. Moreover, since $V$ has a right-adjoint (given by equipping a set $X$ with homs $X(x, y)=\top$ ), it preserves colimits, so we have $V U(\operatorname{colim} I d)=V \operatorname{colim} U=\operatorname{colim} V U$.

From the way that colimits are computed in Set it follows that elements of colim $V U$ are equivalence classes of bisimilarity. Therefore, if the final coalgebra exists, then two states are bisimilar iff they are identified by the unique morphisms into the final coalgebra.

We finish the section with a brief discussion of the notion of similarity from [40, 50], where a relation $R: \mathscr{X} \longrightarrow \mathscr{Y}$ is a $T$-simulation between coalgebras $\xi: \mathscr{X} \longrightarrow T \mathscr{X}$ and $\nu: \mathscr{Y} \longrightarrow T \mathscr{Y}$ if

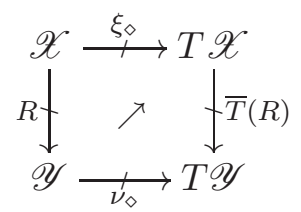

is a lax square. This is a direct generalisation of the notion of bisimulation for Set and [40, 50] show a coinduction theorem stating that the internal hom of the final coalgebra is given by the largest $T$-simulation on the final coalgebra. The following example shows that this coinduction theorem does not capture bisimilarity. Intuitively, the reason is that two-way simulation and bisimilarity are different notions: We can have two elements of the final coalgebra, each simulating the other without being bisimilar.

Example 7.6. Consider $\mathscr{V}=2$, ie $\mathscr{Y}$-cat $=$ Pre, and the functor $T:$ Pre $\longrightarrow$ Pre mapping $\mathscr{X}$ to $2 \times \mathscr{X}$ where $2=\{0,1\}$ is pre-ordered via $0 \leq 1$ and $1 \leq 0$. The final $T$-coalgebra has as elements the streams over 2 , so that two streams are bisimilar iff they are equal. But according to (7.2), any stream can simulate any other.

As explained in [50], the notion of simulation from (7.2) is well-behaved whenever $\bar{T}$ is a lax lifting. 


\section{B. Review of Moss's cover modality $\nabla$ over Set.}

In his seminal paper [36], Moss defined a logic $\mathcal{L}$ for coalgebras for $T:$ Set $\longrightarrow$ Set which is parametric in $T$. First $\mathcal{L}$ is defined inductively by closing under infinite conjunctions and by closing under the functor $T$ itself, so that the logic can be seen as an algebra

$$
(\mathcal{P}+T)(\mathcal{L}) \longrightarrow \mathcal{L}
$$

The component

$$
T \mathcal{L} \stackrel{\nabla}{\longrightarrow} \mathcal{L}
$$

can be understood in more conventional logical terms as stating that $\mathcal{L}$ is closed under $\nabla$, or, more precisely, as stipulating that if $\gamma \in T(\mathcal{L})$ then $\nabla \gamma \in \mathcal{L}$. The semantics of the logic w.r.t. a coalgebra $\xi: X \longrightarrow T X$ and a state $x$ in $X$ is described via a relation

$$
\Vdash_{\xi} \subseteq X \times \mathcal{L}
$$

where we will drop the subscript $\xi$ in the following. The semantics of the operator $\nabla$ is then given using the relation lifting $\bar{T}$ via the inductive clause

$$
x \Vdash \nabla \gamma \Leftrightarrow \bar{T}(\Vdash)(\xi(x), \gamma) .
$$

This can be written as a commuting diagram in the category Rel of sets and relations as follows

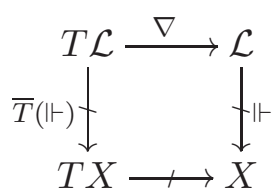

where the lower row is the converse of the graph of $\xi$. One of the basic results of Moss is that the logic is invariant under bisimilarity if $T$ preserves weak pullbacks.

\section{C. The cover modality $\nabla$ over $\mathscr{H}$-cat.}

We are going to redevelop the previous subsection in the enriched setting. First, we take $\Vdash$ from above now to be of the form

$$
\Vdash: \mathscr{X} \otimes \mathcal{L} \longrightarrow \mathscr{V}, \quad \text { that is, } \quad \Vdash: \mathcal{L} \longrightarrow \mathscr{X}^{o p} .
$$

Note that in the case $\mathscr{V}=2$, this conforms with the usual set-up of logics over preorders or posets as eg in [1]. In particular, we have $\varphi \leq \psi \Rightarrow \Vdash(x, \varphi) \leq \Vdash(x, \psi)$ showing that the order on $\mathcal{L}$ behaves semantically as implication and we have $x \leq y \Rightarrow \Vdash(x, \varphi) \leq \Vdash(y, \varphi)$ showing that semantically $\varphi$ behaves like an up-set. These observations continue to make sense in the metric setting, for example, $\mathcal{L}(\varphi, \psi)$ behaves as a $\mathscr{V}$-valued implication which can be seen from that fact that we always have $\Vdash(x, \varphi) \otimes \mathcal{L}(\varphi, \psi) \leq \Vdash(x, \psi)$. For example, in the case of $\mathscr{Y}$-cat $=$ GMet, it says $\Vdash(x, \varphi)+\mathcal{L}(\varphi, \psi) \geq_{\mathrm{R}} \Vdash(x, \psi)$, or more informally, if $\varphi$ is true then $\psi$ cannot deviate from true by more than $\mathcal{L}(\varphi, \psi)$.

Next we want to assume that $\mathcal{L}$ comes equipped with a $\nabla$-operator. As will become clear shortly the ${ }^{o p}$ in (7.4) makes it necessary to take formulas of the kind $\nabla \gamma$ not from $T \mathcal{L}$ but from $T^{\partial} \mathcal{L}$, see Example 6.3. Recall that $T^{\partial}(\mathscr{X})=\left(T\left(\mathscr{X}^{o p}\right)\right)^{o p}$, so that $T$ and $T^{\partial}$ agree on discrete $\mathscr{X}$. So we assume that we have an algebra

$$
T^{\partial} \mathcal{L} \longrightarrow \mathcal{L}
$$


and we define as before in (7.3)

$$
\Vdash(x, \nabla \gamma)=\overline{T^{\partial}}(\Vdash)(\xi(x), \gamma)
$$

which can be expressed diagrammatically as

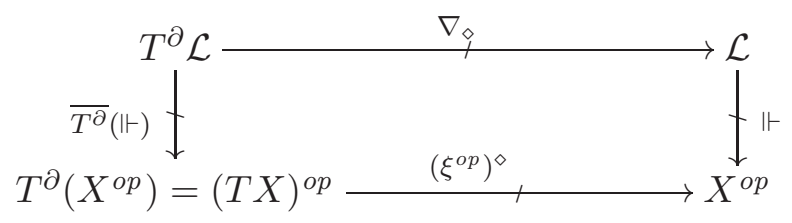

(For the notation $(-)^{\diamond}$ and $\overline{(-)}$ we refer back to Remark 3.6 and Remark 5.12, respectively.)

\section{D. The modality $\nabla$ is invariant under bisimilarity.}

Our next aim is to show that $\nabla$ is invariant under bisimilarity. To this end we use some of the previously developed machinery, in particular from Section 3.A. in order to bring (7.6) into an equivalent but sometimes more useful form (7.7).

Using the adjunction $(-)_{\diamond} \dashv(-)^{\dagger}$ of Proposition 3.8 , to give a relation $\Vdash: \mathcal{L} \longrightarrow \mathscr{X}^{\text {op }}$ is to give to a $\mathscr{V}$-functor $\llbracket \cdot \rrbracket: \mathcal{L} \longrightarrow[\mathscr{X}, \mathscr{V}]$. We consider the $T^{\partial}$-algebra

$$
\left([\mathscr{X}, \mathscr{V}],[\xi, \mathscr{V}] \cdot \delta_{\mathscr{X}^{o p}}\right)
$$

where $\delta_{\mathscr{X}}$ op $: T^{\partial} \mathbb{L} \mathscr{X}^{o p} \longrightarrow \mathbb{L} T^{\partial} \mathscr{X}^{o p}$ is the distributive law of Corollary 3.14 Recall from Remark 3.15 that $\delta_{\mathscr{X}}{ }^{o p}$ corresponds to the relation $\overline{T^{\partial}}\left(\right.$ ev $\left._{\mathscr{X}^{o p}}\right)$, where ev $\mathscr{X}^{o p}:[\mathscr{X}, \mathscr{V}] \longrightarrow \mathscr{X}^{o p}$ is the elementhood relation.

Proposition 7.7. Let $\xi: \mathscr{X} \longrightarrow T \mathscr{X}$ be a coalgebra and $T^{\partial} \mathcal{L} \longrightarrow \mathcal{L}$ an algebra. Consider a relation $\Vdash: \mathcal{L} \longrightarrow \mathscr{X}^{\circ p}$ with $\llbracket \cdot \rrbracket: \mathcal{L} \longrightarrow[\mathscr{X}, \mathscr{V}]$ being the corresponding $\mathscr{V}$-functor given by Proposition 3.8. If $T$ satisfies $B C C$, then the diagram

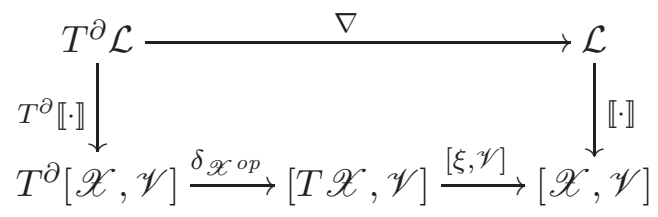

commutes if and only if (17.6) commutes. Moreover, the relation $\Vdash$ is the composition

$$
\mathcal{L} \stackrel{\left(\llbracket \cdot \rrbracket_{\xi}\right)_{\diamond}}{\longrightarrow}[\mathscr{X}, \mathscr{V}] \stackrel{\text { ev } \mathscr{X}_{+}^{o p}}{\longrightarrow} \mathscr{X}^{o p}
$$

Proof. The commutativity of diagram (7.7) amounts to

$$
\begin{aligned}
\Vdash(x, \nabla \alpha) & =\llbracket \nabla \alpha \rrbracket(x) \\
& =[\xi, \mathscr{V}] \delta_{\mathscr{X}}^{o p}\left(T^{\partial} \llbracket \alpha \rrbracket\right)(x) \\
& =\delta_{\mathscr{X}}{ }^{o p}\left(T^{\partial} \llbracket \alpha \rrbracket \xi\right)(x) \\
& =\bar{T}^{\partial}\left(\operatorname{ev}_{\mathscr{X}^{o p}}\right)\left(\xi(x), T^{\partial} \llbracket \alpha \rrbracket\right) .
\end{aligned}
$$


On the other hand, using the fact that $\overline{T^{\partial}}$ preserves composition we have

$$
\begin{aligned}
\overline{T^{\partial}}\left(\mathrm{ev}_{\mathscr{X}^{o p}}\right)\left(\xi(x), T^{\partial} \llbracket \alpha \rrbracket\right) & =\bigvee_{\varphi \in[\mathscr{X}, \mathscr{W}]} \overline{T^{\partial}}\left(\mathrm{ev}_{\mathscr{X}^{o p}}\right)(\xi(x), \varphi) \otimes[\mathscr{X}, \mathscr{V}]\left(\varphi, T^{\partial} \llbracket \alpha \rrbracket\right) \\
& =\bigvee_{\varphi \in[\mathscr{X}, \mathscr{Y}]} \overline{T^{\partial}}\left(\mathrm{ev}_{\mathscr{X}^{o p}}\right)(\xi(x), \varphi) \otimes\left(T^{\partial} \llbracket \cdot \rrbracket\right)_{\diamond}(\varphi, \alpha) \\
& =\bigvee_{\varphi \in[\mathscr{X}, \mathscr{Y}]} \overline{T^{\partial}}\left(\operatorname{ev}_{\mathscr{X}^{o p}}\right)(\xi(x), \varphi) \otimes \overline{T^{\partial}}\left(\llbracket \cdot \rrbracket_{\diamond}\right)(\varphi, \alpha) \\
& =\overline{T^{\partial}}\left(\mathrm{ev}_{\mathscr{X}^{o p}}\right) \cdot \overline{T^{\partial}}(\llbracket \cdot \rrbracket)_{\diamond}(\xi(x), \alpha) \\
& =\overline{T^{\partial}}\left(\mathrm{ev}_{\mathscr{X}}^{o p} \cdot(\mathbb{\llbracket} \cdot \rrbracket)_{\diamond}\right)(\xi(x), \alpha) \\
& =\overline{T^{\partial}}(\Vdash)(\xi(x), \alpha) .
\end{aligned}
$$

To conclude, notice that diagram (7.6) commutes if and only if the equality

$$
\Vdash(x, \nabla \alpha)=\overline{T^{\partial}}(\Vdash)(\xi(x), \alpha)
$$

holds.

As a corollary, we obtain that the semantics of $\nabla$ is invariant under bisimilarity.

Proposition 7.8. If $T$ satisfies $B C C$, then $\nabla$ is invariant under bisimilarity.

Proof. For the purposes of this proof, we do not assume that $\mathcal{L}$ is closed under $\nabla$. Rather we show that if all $\varphi \in \mathcal{L}$ are invariant under bisimilarity, then so are all $\gamma \in T^{\partial} \mathcal{L}$, where the semantics of $\gamma$ wrt a coalgebra $\xi$ is given by the lower-left path of (7.7), that is, by $[\xi, \mathscr{V}] \cdot \delta_{\mathscr{X}}^{o p} \cdot T^{\partial} \llbracket \cdot \rrbracket_{\xi}(\gamma)$

The assumption that all $\varphi \in \mathcal{L}$ are invariant under bisimilarity can be expressed by saying that $\llbracket \cdot \rrbracket_{\xi}: \mathcal{L} \longrightarrow[U \xi, \mathscr{V}]$ is a natural transformation $\llbracket \cdot \rrbracket: \mathcal{L} \longrightarrow[U-, \mathscr{V}]$. This follows immediately from spelling out the naturality condition and comparing with the definition of bisimilarity in Definition [7.4, see also [30]. We thus have to show that

$$
[\xi, \mathscr{V}] \cdot \delta_{\mathscr{X}^{o p}} \cdot T^{\partial} \llbracket \cdot \rrbracket_{\xi}: T^{\partial} \mathcal{L} \longrightarrow[U \xi, \mathscr{V}]
$$

is natural in $\xi$ if

$$
\llbracket \cdot \rrbracket_{\xi}: \mathcal{L} \longrightarrow[U \xi, \mathscr{V}]
$$

is natural in $\xi$. To this end let $f: \mathscr{X} \longrightarrow \mathscr{X}^{\prime}$ be a coalgebra morphism from $\xi: \mathscr{X} \longrightarrow T \mathscr{X}$ to $\xi^{\prime}: \mathscr{X}^{\prime} \longrightarrow T \mathscr{X}^{\prime}$. Consider

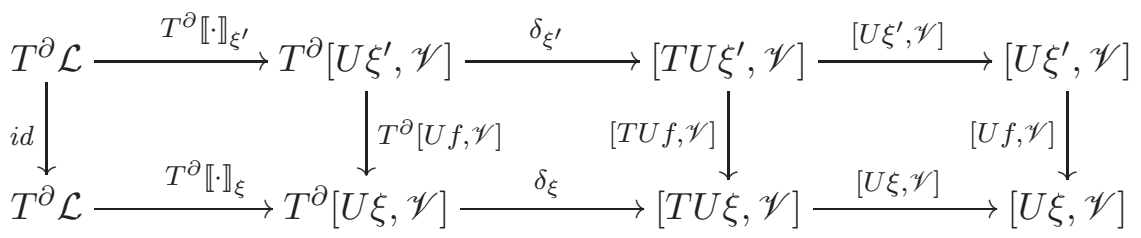

We need to show that the lower row of the diagram is natural in $\xi$. But this is indeed the case. The left square commutes since $\llbracket \cdot \rrbracket_{-}: \mathcal{L} \longrightarrow[U-, \mathscr{V}]$ is natural by assumption, the middle square commutes since $\delta$ is natural (which follows from BCC by Corollary 3.14 and Corollary [5.11), and the right square commutes because $f$ is a coalgebra morphism. 
Remark 7.9. If the initial $T^{\partial}$-algebra exists we denote it by $T^{\partial} \mathcal{L} \stackrel{\nabla}{\longrightarrow} \mathcal{L}$ and take its carrier $\mathcal{L}$ as the collection of all formulas. We can then prove directly that all formulas are invariant under bisimilarity. As in the proposition above, invariance under bisimilarity amounts to naturality of $\llbracket \cdot \rrbracket_{-}: \mathcal{L} \longrightarrow[U-, \mathscr{V}]$, that is, to the commutativity of the right-hand column of

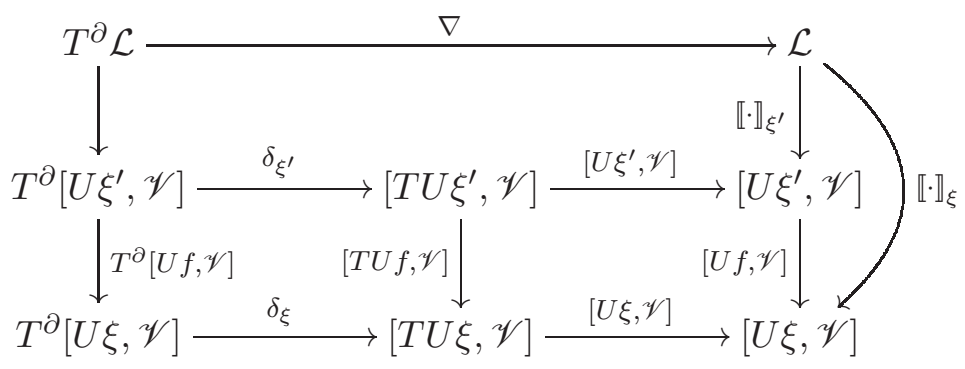

But the right-hand column does indeed commute since homomorphisms out of initial algebras are unique.

\section{E. Examples.}

In the following examples we are going to look at coalgebras

$$
\xi: \mathscr{X} \longrightarrow T \mathscr{X}
$$

for various functors $T$. In each case, we compute the semantics of $\nabla$, which will follow directly from the formulas obtained for the relation liftings in Section 6. In the special case of $\mathscr{V}=2$ the results agree with those obtained in [10].

Remark 7.10. To see the generalisation from 2 to $\mathscr{V}$ we note that in the examples below

(1) the transition structure $\xi$ is $\mathscr{V}$-valued,

(2) the distance between two states of $\mathscr{X}$ is $\mathscr{V}$-valued,

(3) in $\nabla \gamma$, the 'observations' $\gamma$ are weighted in $\mathscr{V}$.

As far as we know, existing work on many-valued modal logic considers only the first of the three items. A useful overview can be found in [13].

For the examples below let us call $\xi$ and $\gamma$ discrete or crisp if they take values only in the subset $\{I, \perp\}$ of the complete lattice $\mathscr{V}$ and let us call them fuzzy otherwise. We also say that $\mathscr{X}$ is discrete if the distance takes values only in $\{I, \perp\}$.

For the remainder of the subsection we assume that a logic $\mathcal{L}$ for $T$-coalgebras $\mathscr{X} \longrightarrow T \mathscr{X}$ is given, either as a module $\Vdash: \mathscr{X} \otimes \mathcal{L} \longrightarrow \mathscr{V}$ or as $\mathscr{V}$-functor $\llbracket \cdot \rrbracket: \mathcal{L} \longrightarrow[\mathscr{X}, \mathscr{V}]$, both forms being equivalent by Proposition 7.7. As pointed out in the proof of Proposition 7.8, the latter form allows us to express the invariance of the logic under bisimilarity by saying that

$$
\llbracket \cdot \rrbracket_{\xi}: \mathcal{L} \longrightarrow[U \xi, \mathscr{V}]
$$

is natural in $\xi$. In each example below, given $\llbracket \cdot \rrbracket_{\xi}$, we will compute

$$
\llbracket \nabla \cdot \rrbracket_{\xi}: T^{\partial} \mathcal{L} \longrightarrow[U \xi, \mathscr{V}] .
$$


Example 7.11. Given a $U$-coalgebra $\xi: \mathscr{X} \longrightarrow \mathbb{U} \mathscr{X}$ and $\gamma \in \mathbb{U}^{\partial}(\mathcal{L})=\mathbb{L}(\mathcal{L})$ we have

$$
\begin{aligned}
\Vdash(x, \nabla \gamma) & =\overline{\mathbb{U}^{\partial}}(\Vdash)(\xi(x), \gamma) \\
& =\bigwedge_{y \in \mathscr{X}}\left[\xi(x)(y), \bigvee_{\varphi \in \mathcal{L}} \Vdash(y, \varphi) \otimes \gamma(\varphi)\right]
\end{aligned}
$$

For discrete $\xi, \gamma, \mathscr{X}$ this becomes as expected

$$
\Vdash(x, \nabla \gamma)=\forall_{y \in \xi(x)} \exists_{\varphi \in \gamma} \Vdash(y, \varphi),
$$

which in turn simplifies, if $\gamma=\mathcal{L}(-, \varphi))^{2}$, to the usual semantics of the $\square$-operator ${ }^{3}$

$$
\Vdash(x, \nabla \mathcal{L}(-, \varphi))=\forall_{y \in \xi(x)} \Vdash(y, \varphi) .
$$

If we allow $\xi$ to be fuzzy, we obtain

$$
\Vdash(x, \nabla \mathcal{L}(-, \varphi))=\bigwedge_{y \in \mathscr{X}}[\xi(x)(y), \Vdash(y, \varphi)]
$$

which is the semantics of $\square$ from [13]. Note that the same formula also accounts for $\mathscr{X}$ which are not discrete, in which case $\xi$ and $\varphi$ need to be non-expanding (a condition which is void for discrete $\mathscr{X})$. Finally, we point out that if the coalgebra structure $\xi: \mathscr{X} \longrightarrow \mathbb{U}$ externalises an internal hom (=identity relation) as in Example 7.3 , then $\Vdash(x, \nabla \mathcal{L}(-, \varphi))=$ $\Vdash(x, \varphi)$ by the Yoneda lemma.

Example 7.12. Given a $\mathbb{L}$-coalgebra $\xi: \mathscr{X} \longrightarrow \mathbb{L} \mathscr{X}$ and $\gamma \in \mathbb{L}^{\partial}(\mathcal{L})=\mathbb{U}(\mathcal{L})$ we have

$$
\begin{aligned}
\Vdash(x, \nabla \gamma) & =\overline{\mathbb{L}^{\partial}}(\Vdash)(\xi(x), \gamma) \\
& =\bigwedge_{\varphi \in \mathcal{L}}\left[\gamma(\varphi), \bigvee_{y \in \mathscr{X}} \Vdash(y, \varphi) \otimes \xi(x)(y)\right]
\end{aligned}
$$

For discrete $\xi, \gamma, \mathscr{X}$ this becomes as expected

$$
\Vdash(x, \nabla \gamma)=\forall_{\varphi \in \gamma} \exists_{y \in \xi(x)} \Vdash(y, \varphi),
$$

which in turn simplifies, if $\gamma=\mathcal{L}(\varphi,-)$, to the usual semantics of the $\diamond$-operator

$$
\Vdash(x, \nabla \mathcal{L}(\varphi,-))=\exists_{y \in \xi(x)} \Vdash(y, \varphi) .
$$

If we allow $\xi$ to be fuzzy, we obtain

$$
\Vdash(x, \nabla \mathcal{L}(\varphi,-))=\bigvee_{y \in \mathscr{X}} \Vdash(y, \varphi) \otimes \xi(x)(y)
$$

which is the semantics of $\diamond$ from [13]. Again the same formula also accounts for non-discrete $\mathscr{X}$.

Example 7.13. Consider a quantale $\mathscr{V}$ such that $\otimes=\wedge$. Given a P-coalgebra $\xi: \mathscr{X} \longrightarrow$ $\mathbb{P} \mathscr{X}$, the $\nabla$-semantics wrt $\xi$ is given as follows. Observe that $\mathbb{P}=\mathbb{P}^{\partial}$, thus $\mathcal{L}$ is a $\mathbb{P}$-algebra. For every $x \in \mathscr{X}$ and $\gamma \in \mathbb{P}^{\partial}(\mathcal{L})=\mathbb{P}(\mathcal{L})$ we have

$$
\begin{aligned}
\Vdash(x, \nabla \gamma) & =\overline{\mathbb{P}^{\partial}}(\Vdash)(\xi(x), \gamma) \\
& =\bigwedge_{y \in \mathscr{X}}\left[\xi(x)(y), \bigvee_{\varphi \in \mathcal{L}} \Vdash(y, \varphi) \otimes \gamma(\varphi)\right] \otimes \bigwedge_{\varphi \in \mathcal{L}}\left[\gamma(\varphi), \bigvee_{y \in \mathscr{X}} \Vdash(y, \varphi) \otimes \xi(x)(y)\right]
\end{aligned}
$$

\footnotetext{
${ }^{2} \mathcal{L}(-, \varphi)$ can be thought as the lowerset spanned by $\varphi$.

${ }^{3}$ Over Set and for $T$ the powerset functor, we have $\nabla\{\varphi\}=\square \varphi \wedge \diamond \varphi$. This is different here because $\mathrm{U}$ is only "one half" of the power functor.
} 
For discrete $\xi, \gamma, \mathscr{X}$ this becomes as expected

$$
\Vdash(x, \nabla \gamma)=\forall_{y \in \xi(x)} \exists_{\varphi \in \gamma} \Vdash(y, \varphi) \wedge \forall_{\varphi \in \gamma} \exists_{y \in \xi(x)} \Vdash(y, \varphi)
$$

which coincides with the usual semantics of $\nabla$ for the powerset functor. If we keep $\gamma$ discrete, but allow the formulas $\varphi \in \gamma$ to be fuzzy, then we obtain, in the case of generalised ultrametric spaces, ie $\mathscr{Y}$-cat $=$ GUlt,

$$
\Vdash(x, \nabla \gamma)=\max \left(\sup _{y \in \xi(x)} \inf _{\varphi \in \gamma} \Vdash(y, \varphi), \quad \sup _{\varphi \in \gamma} \inf _{y \in \xi(x)} \Vdash(y, \varphi)\right)
$$

which was proposed as a many-valued semantics of $\nabla$ in [17] (with the difference that in this paper the order on $[0 ; 1]$ is reversed).

\section{Conclusion}

The emphasis of the work reported here has been on a category theoretic study of relation liftings in the setting of categories enriched over a complete commutative quantale. In particular, it was shown that the familiar characterisations known for functors $T$ : Set $\longrightarrow$ Set can be extended to functors $T: \mathscr{Y}$-cat $\longrightarrow \mathscr{V}$-cat. First, we proved (Corollary 3.14) that the relation lifting $\bar{T}: \mathscr{V}$-mod $\longrightarrow \mathscr{Y}$-mod is functorial iff there is a distributive law

$$
T \cdot \mathbb{L} \longrightarrow \mathbb{L} \cdot T \text {. }
$$

This parallels the known result from Set where the "lower-set functor" $\mathbb{~ r e p l a c e s ~ t h e ~ c o v a r i - ~}$ ant powerset functor. We also proved (Corollary 5.11) that the relation lifting $\bar{T}: \mathscr{V}$-mod $\longrightarrow$ $\mathscr{V}$-mod is functorial iff $T$ satisfies the Beck-Chevalley Condition, that is, if $T$ preserves exact squares, which replaces the condition from Set that $T$ weakly preserves pullbacks.

One motivation of our investigations stems from coalgebraic logic. In particular, we could show that once we have the relation lifting working, the semantics of Moss's cover modality $\nabla$ could be defined essentially as over Set. Moreover, although computations get more complicated, the machinery developed here works smoothly enough and a number of concrete examples have been worked out.

An exciting direction for future work is to see whether our insights can be applied to give a systematic account of many-valued modal logics. Whereas many-valued logics have a long tradition and a deep and beautiful algebraic theory, see eg [16], much less work has been done on extensions of these logics by modal operators. It will be interesting to see how far we can go with transferring results from coalgebraic logic over Set to $\mathscr{K}$-cat, thus developing many-valued coalgebraic logic.

\section{REFERENCES}

[1] S. Abramsky and A. Jung, Domain theory, In: Handbook of Logic in Computer Science (1994), 1-168. Clarendon Press.

[2] P. Aczel, N. P. Mendler, A final coalgebra theorem, LNCS 389, Springer 1989, 357-365.

[3] J. Adámek, R. El Bashir, M. Sobral and J. Velebil, On functors that are lax epimorphisms, Theory Appl. Categ. 8.20 (2001), 509-521.

[4] A. Balan and A. Kurz, On coalgebras over algebras, Theor. Comput. Sci. 412.38 (2011), 4989-5005.

[5] A. Baltag, A logic for coalgebraic simulation, Electron. Notes Theor. Comput. Sci. 33 (2000), 41-60.

[6] J. de Bakker and E. de Vink, Control flow semantics, MIT Press, 1996.

[7] M. Barr, Relational algebras, Lecture Notes in Mathematics 137, Springer 1970, 39-55. 
[8] R. El Bashir and J. Velebil, Reflective and coreflective subcategories of presheaves, Theory Appl. Categ. 10.16 (2002), 410-423.

[9] J. Bénabou, Critères de représentabilité des foncteurs, C. R. Math. Acad. Sci. Paris 260 (1965), $752-755$.

[10] M. Bílková, A. Kurz, D. Petrişan and J. Velebil, Relation liftings on preorders and posets, LNCS 6859, Springer 2011, 115-129.

[11] M. M. Bonsangue, F. van Breugel and J. J. M. M. Rutten, Generalized metric spaces: completion, topology, and powerdomains via the Yoneda embedding, CWI Report CS-R9636, Amsterdam, 1996.

[12] M. M. Bonsangue, F. van Breugel and J. J. M. M. Rutten, Generalized metric spaces: completion, topology, and powerdomains via the Yoneda embedding, Theor. Comput. Sci. 193 (1998), 1-51.

[13] F. Bou and F. Esteva and L. Godo and R. Rodríguez, On the minimum many-valued modal logic over a finite residuated lattice, J. Log. Comput. 21 (2011), 739-790.

[14] A. Carboni, S. Johnson, R. Street and D. Verity, Modulated bicategories, J. Pure Appl. Algebra 94 (1994), 229-282

[15] A. Carboni, G. M. Kelly and R. J. Wood, A 2-categorical approach to change of base and geometric morphisms I, Cahiers de Top. et Géom. Diff. XXXII (1991), 47-95.

[16] R. Cignoli, I. D’Ottaviano, and D. Mundici, Algebraic Foundations of Many-valued Reasoning, Kluwer 2000.

[17] M. Dostál and A. Kurz, Some observations on the many-valued semantics of the cover modality, Technical report, Feb 2012.

[18] B. Flagg and R. Kopperman, Continuity spaces: reconciling domains and metric spaces, Theor. Comput. Sci. 177.1 (1997), 111-138.

[19] R. Guitart, Relations et carrés exacts, Ann. Sci. Math. Québec IV.2 (1980), 103-125.

[20] P. Hájek, Metamathematics of fuzzy logic, Kluwer 1998.

[21] C. Hermida, A categorical outlook on relational modalities and simulations, Inf. Comput., 209.12 (2011), 1505-1517.

[22] C. Hermida and B. Jacobs, Structural induction and coinduction in a fibrational setting, Inform. and Comput. 145.2 (1998), 107-152.

[23] D. Hofmann and C. D. Reis, Probabilistic metric spaces as enriched categories, http://front.math.ucdavis.edu/1201.1161

[24] J. Hughes and B. Jacobs, Simulations in coalgebra, Theor. Comput. Sci. 327 (2004), 71-108.

[25] G. M. Kelly, Basic concepts of enriched category theory, London Math. Soc. Lecture Notes Series 64, Cambridge Univ. Press, 1982, Repr. Theory Appl. Categ. 10 (2005), 1-136.

[26] G. M. Kelly, Elementary observations on 2-categorical limits, Bull. Austral. Math. Soc. 39 (1989), 301-317.

[27] B. Klin, An abstract coalgebraic approach to process equivalence for well-behaved operational semantics, PhD Thesis, University of Aarhus, 2004.

[28] A. Kock, Monads for which structures are adjoint to units, J. Pure Appl. Algebra 104.1 (1995), 41-59.

[29] C. Kupke, A. Kurz and Y. Venema, Completeness of the finitary Moss logic, in Advances in Modal Logic 2008, 193-217.

[30] A. Kurz and J. Rosický. Operations and equations for coalgebras. Math. Structures Comput. Sci., 15:149-166, 2005.

[31] W. Lawvere, Metric spaces, generalized logic and closed categories, Repr. Theory Appl. Categ. 1 (2002), 1-37.

[32] P. B. Levy, Similarity quotients as final coalgebras, LNCS 6604, Springer 2011, 27-41.

[33] S. Mac Lane, Categories for the working mathematician, Springer, 1998.

[34] F. Marmolejo, Distributive laws for pseudomonads, Theor. Appl. Categ. 5.5 (1999), 91-147.

[35] F. Marmolejo, R. D. Rosebrugh and R. J. Wood, Duality for CCD lattices, Theor. Appl. Categ. 22.1 (2009), 1-23.

[36] L. Moss, Coalgebraic logic, Ann. Pure Appl. Logic 96 (1999), 277-317.

[37] nLab page: Codiscrete cofibration, http://ncatlab.org/nlab/show/codiscrete+cofibration, revised on September 11, 2011 03:31:06 by Mike Shulman

[38] A. Palmigiano, A coalgebraic view on positive modal logic, Theor. Comput. Sci. 327 (2004), 175-195.

[39] E. Riehl, Two-sided discrete fibrations in 2-categories and bicategories, preprint, available at http://www.math.uchicago.edu/ eriehl/ 
[40] J. J. M. M. Rutten, Relators and metric bisimulations, Electron. Notes Theor. Comput. Sci. 11 (1998), $252-258$.

[41] J. J. M. M. Rutten. Universal coalgebra: A theory of systems. Theor. Comput. Sci., 249:3-80, 2000.

[42] S. Staton, Relating coalgebraic notions of bisimulation, Logical Methods in Computer Science, 7 (2011).

[43] R. Street, Cauchy characterization of enriched categories, Rendiconti del Seminario Matematico e Fisico di Milano 51 (1981), 217-233, also available as Repr. Theory Appl. Categ 4 (2004), 1-16.

[44] R. Street, Characterizations of bicategories of stacks, Lecture Notes in Mathematics 962, Springer 1982, 282-291.

[45] R. Street, Fibrations in bicategories, Cahiers de Top. et Géom. Diff. XXI.2 (1980), 111-159

[46] V. Trnková, General theory of relational automata, Fund. Inform. 3 (1980), 189-233.

[47] J. Velebil and A. Kurz, Equational presentations of functors and monads, Math. Struct. Comput. Sci. 21.2 (2011), 363-381

[48] Y. Venema, Automata and fixed point logics: A coalgebraic perspective, Inform. and Comput. 204.4 (2006), 637-678.

[49] J. Worrell, On coalgebras and final semantics, PhD Thesis, Oxford University Computing Laboratory, 2000.

[50] J. Worrell, Coinduction for recursive data types: partial order, metric spaces and $\Omega$-categories, Electron. Notes Theor. Comput. Sci. 33 (2000), 367-386. 